\title{
Front Matter: Volume 7731
}

, "Front Matter: Volume 7731," Proc. SPIE 7731, Space Telescopes and Instrumentation 2010: Optical, Infrared, and Millimeter Wave, 773101 (31 August 2010); doi: 10.1117/12.871349

SDIE Event: SPIE Astronomical Telescopes + Instrumentation, 2010, San Diego, California, United States 


\section{PROCEEDINGS OF SPIE}

\section{Space Telescopes and Instrumentation 2010: Optical, Infrared, and Millimeter Wave}

Jacobus M. Oschmann, Jr.

Mark C. Clampin

Howard A. MacEwen

Editors

27 June-2 July 2010

San Diego, California, United States

Sponsored by

SPIE

Cooperating Organizations

American Astronomical Society (United States) - Association of Universities for Research in Astronomy, Inc. (United States) - Astronomical Society of Japan (Japan) - Atacama Large Millimeter/Submillimeter Array • Ball Aerospace \& Technologies Corporation (United States) Canadian Astronomical Society (CASCA) (Canada) - Commissariat á l'Energie Atomique (France) • European Astronomical Society (Switzerland) • ESO_European Organisation for Astronomical Research in the Southern Hemisphere (Germany) • Japan Aerospace Exploration Agency (Japan) • Jet Propulsion Laboratory (United States) • NASA Goddard Space Flight Center (United States) • National Astronomical Observatory Japan (Japan) National Radio Astronomy Observatory - SOFIA-Stratospheric Observatory for Infrared Astronomy (United States) - Thirty Meter Telescope Project (United States) • W. M. Keck Observatory (United States)

Published by

SPIE

Volume 7731 
The papers included in this volume were part of the technical conference cited on the cover and title page. Papers were selected and subject to review by the editors and conference program committee. Some conference presentations may not be available for publication. The papers published in these proceedings reflect the work and thoughts of the authors and are published herein as submitted. The publisher is not responsible for the validity of the information or for any outcomes resulting from reliance thereon.

Please use the following format to cite material from this book:

Author(s), "Title of Paper," in Space Telescopes and Instrumentation 2010: Optical, Infrared, and Millimeter Wave, edited by Jacobus M. Oschmann, Jr., Mark C. Clampin, Howard A. MacEwen, Proceedings of SPIE Vol. 7731 (SPIE, Bellingham, WA, 2010) Article CID Number.

ISSN 0277-786X

ISBN 9780819482211

Published by

SPIE

P.O. Box 10, Bellingham, Washington 98227-0010 USA

Telephone +1 3606763290 (Pacific Time) · Fax +1 3606471445

SPIE.org

Copyright (@ 2010, Society of Photo-Optical Instrumentation Engineers

Copying of material in this book for internal or personal use, or for the internal or personal use of specific clients, beyond the fair use provisions granted by the U.S. Copyright Law is authorized by SPIE subject to payment of copying fees. The Transactional Reporting Service base fee for this volume is $\$ 18.00$ per article (or portion thereof), which should be paid directly to the Copyright Clearance Center (CCC), 222 Rosewood Drive, Danvers, MA 01923. Payment may also be made electronically through CCC Online at copyright.com. Other copying for republication, resale, advertising or promotion, or any form of systematic or multiple reproduction of any material in this book is prohibited except with permission in writing from the publisher. The CCC fee code is 0277-786X/10/\$18.00.

Printed in the United States of America.

Publication of record for individual papers is online in the SPIE Digital Library.

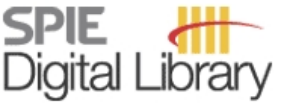

SPIEDigitalLibrary.org

Paper Numbering: Proceedings of SPIE follow an e-First publication model, with papers published first online and then in print and on CD-ROM. Papers are published as they are submitted and meet publication criteria. A unique, consistent, permanent citation identifier (CID) number is assigned to each article at the time of the first publication. Utilization of CIDs allows articles to be fully citable as soon they are published online, and connects the same identifier to all online, print, and electronic versions of the publication. SPIE uses a six-digit CID article numbering system in which:

- The first four digits correspond to the SPIE volume number.

- The last two digits indicate publication order within the volume using a Base 36 numbering system employing both numerals and letters. These two-number sets start with 00, 01, 02, 03, 04 , 05, 06, 07, 08, 09, OA, OB ... 0Z, followed by 10-1Z, 20-2Z, etc.

The CID number appears on each page of the manuscript. The complete citation is used on the first page, and an abbreviated version on subsequent pages. Numbers in the index correspond to the last two digits of the six-digit CID number. 


\title{
Contents
}

\section{Part One}

\author{
xxxi Conference Committee \\ xxxV Introduction \\ xxxix Unknowns and unknown unknowns: from dark sky to dark matter and dark energy \\ (Plenary Paper) [7733-501] \\ Y. Suto, The Univ. of Tokyo (Japan) \\ li Optical synoptic telescopes: new science frontiers (Plenary Paper) [7733-502] \\ J. A. Tyson, Univ. of California, Davis (United States)
}

\section{SESSION 1 STRATEGIES}

$773102 \quad K e y$ enabling technologies for the next generation of space telescopes [7731-01]

C. F. Lillie, R. S. Polidan, D. R. Dailey, Northrop Grumman Aerospace Systems (United States)

773103 Early results from NASA's assessment of satellite servicing [7731-02]

B. B. Reed, J. A. Townsend, H. A. Thronson, Jr., M. Ahmed, A. O. Whipple, W. R. Oegerle, NASA Goddard Space Flight Ctr. (United States)

773104 Preliminary multivariable cost model for space telescopes [7731-03] H. P. Stahl, NASA Marshall Space Flight Ctr. (United States); T. Henrichs, Middle Tennessee State Univ. (United States)

\section{SESSION 2 JWST I}

773107 Overview of the James Webb Space Telescope Observatory [7731-06]

M. Clampin, NASA Goddard Space Flight Ctr. (United States); E. P. Smith, NASA Headquarters (United States)

773108 Status of the James Webb Space Telescope integrated science instrument module system [7731-07]

M. A. Greenhouse, M. P. Drury, J. L. Dunn, S. D. Glazer, E. Greville, G. Henegar, E. L. Johnson, R. Lundquist, J. C. McCloskey, R. G. Ohl IV, R. A. Rashford, M. F. Voyton, NASA Goddard Space Flight Ctr. (United States)

\section{SESSION 3 JWST II}

7731 OA Results, status, and plans for the James Webb Space Telescope optical telescope element [7731-09]

L. D. Feinberg, R. Keski-Kuha, NASA Goddard Space Flight Ctr. (United States); C. Atkinson,

S. C. Texter, Northrop Grumman Aerospace Systems (United States) 
7731 OB Optical performance for the actively controlled James Webb Space Telescope [7731-10] P. A. Lightsey, D. Chaney, B. Gallagher, B. Brown, K. Smith, J. Lewis, A. Barto, S. Knight, S. Acton, C. Stewart, N. Siegel, Ball Aerospace \& Technologies Corp. (United States)

7731 OC NIRCam: development and testing of the JWST near-infrared camera [7731-11] T. Greene, NASA Ames Research Ctr. (United States); C. Beichman, California Institute of Technology (United States); M. Gully-Santiago, D. Jaffe, The Univ. of Texas at Austin (United States); D. Kelly, Steward Observatory, The Univ. of Arizona (United States); J. Krist, Jet Propulsion Lab. (United States); M. Rieke, Steward Observatory, The Univ. of Arizona (United States); E. H. Smith, Lockheed Martin Advanced Technology Ctr. (United States)

7731 OD Status of the NIRSpec instrument [7731-12]

S. M. Birkmann, T. Böker, P. Jakobsen, G. de Marchi, M. Sirianni, G. Bagnasco, P. L. Jensen, M. B. J. te Plate, P. Rumler, J.-C. Salvignol, P. Strada, European Space Research and Technology Ctr. (Netherlands); M. Kolm, X. Gnata, K. Honnen, J. Köhler, R. Lemke, M. Maschmann, M. Melf, J.-F. Pittet, EADS Astrium GmbH (Germany); P. Ferruit, B. Dorner, Univ. de Lyon (France), Observatoire de Lyon, Univ. Lyon 1 (France), Ctr. de Recherche Astrophysique de Lyon, CNRS (France), and Ecole Normale Supérieure de Lyon (France)

7731 OE Progress with the design and development of MIRI, the mid-IR instrument for JWST [7731-13] G. S. Wright, UK Astronomy Technology Ctr. (United Kingdom); G. Rieke, Steward Observatory, The Univ. of Arizona (United States); T. Boeker, European Space Research and Technology Ctr. (Netherlands); L. Colina, Consejo Superior de Investigaciones Científicas (Spain); E. van Dishoeck, Leiden Observatory, Leiden Univ. (Netherlands); P. Driggers, NASA Goddard Space Flight Ctr. (United States); S. Friedman, Space Telescope Science Institute (United States); A. Glasse, UK Astronomy Technology Ctr. (United Kingdom); G. Goodson, Jet Propulsion Lab. (United States); T. Greene, NASA Ames Research Ctr. (United States); M. Guedel, Univ. of Vienna (Austria); T. Henning, Max-Planck-Institut für Astronomie (Germany); P.-O. Lagage, Service d'Astrophysique, CEA (France); J. Lorenzo-Alvarez, European Space Research and Technology Ctr. (Netherlands); M. Meixner, Space Telescope Science Institute (United States); H. Norgaard-Nielsen, Danish Space Research Institute (Denmark); G. Olofsson, Stockholm Observatory (Sweden); T. Ray, The Dublin Institute for Advanced Studies (Ireland); M. Ressler, K. Sukhatme, Jet Propulsion Lab. (United States); J. Thatcher, EADS Astrium Ltd. (United Kingdom); C. Waelkens, Instituut voor Sterrenkunde, Katholieke Univ. Leuven (Belgium); D. Wright, EADS Astrium Ltd. (United Kingdom)

7731 OF The JWST tunable filter imager (TFI) [7731-14]

R. Doyon, Univ. de Montréal (Canada); J. Hutchings, Herzberg Institute of Astrophysics, National Research Council Canada (Canada); N. Rowlands, C. E. Evans, E. Greenberg, C. Haley, A. D. Scott, D. Touahri, COM DEV Canada (Canada); M. Beaulieu, D. Lafrenière, Univ. de Montréal (Canada); R. Abraham, Univ. of Toronto (Canada); E. Barton, Univ. of California, Irvine (United States); P. Chayer, Space Telescope Science Institute (Canada); L. Ferrarese, Herzberg Institute of Astrophysics, National Research Council Canada (Canada); A. W. Fullerton, Space Telescope Science Institute (Canada); R. Jayawardhana, Univ. of Toronto (Canada); D. Johnstone, Herzberg Institute of Astrophysics, National Research Council Canada (Canada); A. Martel, Space Telescope Science Institute (Canada); A. W. M. R. Meyer, Institute for Astronomy, ETH Zurich (Germany); J. Pipher, Univ. of Rochester (United States); K. Saad, Canadian Space Agency (Canada); M. Sawicki, Saint Mary's Univ. (Canada); A. Sivaramakrishnan, American Museum of Natural History (United States); K. Volk, Space Telescope Science Institute (Canada) 
7731 ol Successful production of the engineering development unit (EDU) primary mirror segment and flight unit tertiary mirror for JWST [7731-17]

A. Arneson, C. Alongi, R. Bernier, E. Boese, J. Daniel, L. Dettmann, R. Garfield, H. Glatzel, J. Kincade, P. Johnson, A. Lee, A. Magruder, A. Patel, M. Seilonen, G. Surges, L-3 Communications Tinsley Labs. Inc. (United States); M. Bergeland, R. J. Brown, B. Gallagher, Ball Aerospace \& Technologies Corp. (United States); A. McKay, Northrop Grumman Aerospace Systems (United States); L. M. Cohen, Smithsonian Astrophysical Observatory (United States)

7731 0J Optical performance of the JWST/MIRI flight model: characterization of the point spread function at high resolution [7731-18] P. Guillard, Spitzer Science Ctr., California Institute of Technology (United States) and Institut d'Astrophysique Spatiale, CNRS, Univ. Paris Sud 11 (France); T. Rodet, Lab. des Signaux et Systèmes, CNRS, Univ. Paris Sud 11 (France); S. Ronayette, J. Amiaux, Lab. d'Astrophysique, Instrumentation et Modélisation, CEA/IRFU, Univ. Paris-Diderot, CNRS/INSU, CEA Saclay (France); A. Abergel, Institut d'Astrophysique Spatiale, CNRS, Univ. Paris 11 (France); V. Moreau, J. L. Augueres, A. Bensalem, T. Orduna, C. Nehmé, A. R. Belu, E. Pantin, P.-O. Lagage, Lab. d'Astrophysique, Instrumentation et Modélisation, CEA/IRFU, Univ. Paris-Diderot, CNRS/INSU, CEA Saclay (France); Y. Longval, Institut d'Astrophysique Spatiale, CNRS, Univ. Paris 11 (France); A. C. H. Glasse, UK Astronomy Technology Ctr. (United Kingdom); P. Bouchet, Lab. d'Astrophysique, Instrumentation et Modélisation, CEA/IRFU, Univ. Paris-Diderot, CNRS/INSU, CEA Saclay (France); C. Cavarroc, Institut d'Astrophysique Spatiale, CNRS, Univ. Paris 11 (France); D. Dubreuil, Lab. d'Astrophysique, Instrumentation et Modélisation, CEA/IRFU, Univ. Paris-Diderot, CNRS/INSU, CEA Saclay (France); S. Kendrew, Leiden Univ. (Netherlands)

7731 OK The throughput and sensitivity of the JWST mid-infrared instrument [7731-19]

A. C. H. Glasse, UK Astronomy Technology Ctr. (United Kingdom); E. Bauwens, Instituut voor Sterrenkunde, Katholieke Univ. Leuven (Belgium); J. Bouwman, Ö. Detre, Max-Planck-Institut für Astronomie (Germany); S. Fischer, M. Garcia-Marin, Univ. zu Köln (Germany); K. Justannont, Onsala Space Observatory, Chalmers Univ. of Technology (Sweden); A. Labiano, European Space Agency (Spain); T. Nakos, Sterrenkundig Observatorium, Univ. Gent (Belgium); M. Ressler, Jet Propulsion Lab. (United States); G. Rieke, Steward Observatory, The Univ. of Arizona (United States); S. Scheithaver, Max-Planck-Institut für Astronomie (Germany); M. Wells, G. S. Wright, UK Astronomy Technology Ctr. (United Kingdom)

7731 OL Testing a critical stray light path of the James Webb Space Telescope [7731-20]

T. Whitman, ITT Geospatial Systems (United States)

\section{SESSION 5 AKARI/SPITZER}

7731 OM AKARI warm mission [7731-21]

T. Onaka, The Univ. of Tokyo (Japan); H. Matsuhara, T. Wada, Japan Aerospace Exploration Agency (Japan); D. Ishihara, Nagoya Univ. (Japan); Y. Ita, Astronomical Institute, Tohoku Univ. (Japan); Y. Ohyama, Institute of Astronomy and Astrophysics, Academica Sinica (Taiwan); T. Ootsubo, Astronomical Institute, Tohoku Univ. (Japan); S. Oyabu, Subaru Telescope, National Astronomical Observatory of Japan (Japan); I. Sakon, T. Shimonishi, The Univ. of Tokyo (Japan); S. Takita, Japan Aerospace Exploration Agency (Japan); T. Tanabè, 
Institute of Astronomy, The Univ. of Tokyo (Japan); F. Usui, H. Murakami, Japan Aerospace Exploration Agency (Japan)

7731 ON Calibration and data quality of warm IRAC [7731-22]

S. J. Carey, J. A. Surace, W. J. Glaccum, J. Ingalls, J. Krick, Spitzer Science Ctr., California Institute of Technology (United States); M. Lacy, Spitzer Science Ctr., California Institute of Technology (United States) and National Radio Astronomy Observatory (United States); P. Lowrance, S. Laine, J. O'Linger, J. R. Stauffer, Spitzer Science Ctr., California Institute of Technology (United States); S. P. Willner, J. L. Hora, Harvard-Smithsonian Ctr. for Astrophysics (United States); W. F. Hoffmann, Steward Observatory, The Univ. of Arizona (United States); M. L. N. Ashby, J.-S. Huang, Harvard-Smithsonian Ctr. for Astrophysics (United States); M. Marengo, Harvard-Smithsonian Ctr. for Astrophysics (United States) and lowa State Univ. (United States); M. Pahre, Z. Wang, Harvard-Smithsonian Ctr. for Astrophysics (United States); M. Werner, Jet Propulsion Lab. (United States); G. G. Fazio, Harvard-Smithsonian Ctr. for Astrophysics (United States)

\section{SESSION 6 SPICA I}

773100 The next-generation space infrared astronomy mission SPICA [7731-23]

T. Nakagawa, Japan Aerospace Exploration Agency (Japan)

7731 OP System requirements and design concept of the SPICA Mission [7731-24]

N. Takahashi, T. Nakagawa, H. Murakami, H. Matsuhara, H. Sugita, T. Yamawaki, Japan Aerospace Exploration Agency (Japan)

$77310 Q$ Conceptual design for the mid-infrared medium-resolution Echelle spectrometer (MIRMES) on the SPICA Mission [7731-25]

I. Sakon, The Univ. of Tokyo (Japan); Y. Ikeda, Photocoding (Japan) and Kyoto-Sangyo Univ. (Japan); N. Fujishiro, Cybernet Systems Co. Ltd. (Japan); H. Kataza, Japan Aerospace Exploration Agency (Japan); Y. Okada, Univ. zu Köln (Germany); T. Onaka, The Univ. of Tokyo (Japan)

\section{SESSION 7 SPICA II}

7731 OS The background-limited infrared-submillimeter spectrograph (BLISS) for SPICA: a design study [7731-27]

C. M. Bradford, J. Bock, W. Holmes, M. Kenyon, A. Beyer, M. Werner, M. Rud, T. Prouvé, P. Echternach, Jet Propulsion Lab. (United States); K. Irwin, S. Cho, National Institute of Standards and Technology (United States); M. Harwit, G. Stacey, Cornell Univ. (United States); G. Helou, L. Armus, P. Appleton, California Institute of Technology (United States); J. D. Smith, The Univ. of Toledo (United States); U. Gorti, NASA Ames Research Ctr. (United States); G. Rieke, E. Egami, The Univ. of Arizona (United States); D. Lester, The Univ. of Texas at Austin (United States); J. Glenn, Univ. of Colorado at Boulder (United States); M. Malkan, Univ. of California, Los Angeles (United States); D. Dale, Univ. of Wyoming (United States)

7731 OT WISPIR: a wide-field imaging spectrograph for the infrared for the SPICA Observatory [7731-28]

D. J. Benford, NASA Goddard Space Flight Ctr. (United States); L. G. Mundy, Univ. of Maryland, College Park (United States) 
7731 OU Mid-infrared camera without lens (MIRACLE) for SPICA [7731-29]

T. Wada, H. Kataza, Japan Aerospace Exploration Agency (Japan)

7731 OV Optical testing activities for the SPICA telescope [7731-30]

H. Kaneda, Nagoya Univ. (Japan); T. Nakagawa, K. Enya, Y. Tange, T. Imai, H. Katayama, M. Suganuma, M. Naitoh, K. Maruyama, Japan Aerospace Exploration Agency (Japan); T. Onaka, The Univ. of Tokyo (Japan); Y. Kiriyama, T. Mori, A. Takahashi, Nagoya Univ. (Japan)

\section{SESSION 8 WISE}

7731 OX Pre-launch characterization of the WISE payload [7731-32]

H. Latvakoski, J. Cardon, M. Larsen, J. Elwell, Space Dynamics Lab., Utah State Univ. (United States)

7731 OY Hardware results for the Wide-field Infrared Survey Explorer (WISE) telescope and scanner [7731-33]

M. Schwalm, A. Akerstrom, M. Barry, J. Guregian, L-3 Integrated Optical Systems SSG (United States); M. Larsen, Space Dynamics Lab., Utah State Univ. (United States); P. Laquidara,

J. Regan, V. Ugolini, L-3 Integrated Optical System SSG (United States)

\section{SESSION 9 HUBBLE}

$77310 Z$ On-orbit performance of HST Wide Field Camera 3 (Invited Paper) [7731-34]

J. W. MacKenty, Space Telescope Science Institute (United States); R. A. Kimble, NASA Goddard Space Flight Ctr. (United States); R. W. O'Connell, Univ. of Virginia (United States); J. A. Townsend, NASA Goddard Space Flight Ctr. (United States)

773111 Feasibility of exoplanet coronagraphy with the Hubble Space Telescope [7731-36] R. G. Lyon, NASA Goddard Space Flight Ctr. (United States); R. A. Woodruff, Lockheed Martin Space Systems Co. (United States); R. Brown, Space Telescope Science Institute (United States); M. C. Noecker, Ball Aerospace \& Technologies Corp. (United States); E. Cheng, Conceptual Analytics, LLC (United States)

\section{SESSION 10 HERSCHEL}

773116 In-orbit performance of the Herschel/SPIRE imaging Fourier transform spectrometer [7731-41]

D. A. Naylor, Institute for Space Imaging Science, Univ. of Lethbridge (Canada); J.-P. Baluteau, Lab. d'Astrophysique de Marseille (France); M. J. Barlow, Univ. College London (United Kingdom); D. Benielli, Lab. d'Astrophysique de Marseille (France); M. Ferlet, Rutherford Appleton Lab. (United Kingdom); T. R. Fulton, Blue Sky Spectroscopy Inc. (Canada); M. J. Griffin, Cardiff Univ. (United Kingdom); T. Grundy, Rutherford Appleton Lab. (United Kingdom); P. Imhof, Blue Sky Spectroscopy Inc. (Canada); S. Jones, Institute for Space Imaging Science, Univ. of Lethbridge (Canada); K. King, S. J. Leeks, T. L. Lim, Rutherford Appleton Lab. (United Kingdom); N. Lu, California Institute of Technology (United States); G. Makiwa, E. T. Polehampton, Institute for Space Imaging Science, Univ. of Lethbridge (Canada); G. Savini, Univ. College London (United Kingdom); S. D. Sidher, Rutherford Appleton Lab. (United Kingdom); L. D. Spencer, Cardiff Univ. (United Kingdom); 
C. Surace, Lab. d'Astrophysique de Marseille (France); B. M. Swinyard, Rutherford Appleton Lab. (United Kingdom); R. Wesson, Lab. d'Astrophysique de Marseille (France)

\section{SESSION 11 KEPLER}

773117 Kepler instrument performance: an in-flight update [7731-42]

D. A. Caldwell, J. E. Van Cleve, J. M. Jenkins, NASA Ames Research Ctr. (United States); V.S. Argabright, Ball Aerospace \& Technologies Corp. (United States); J. J. Kolodziejczak, NASA Marshall Space Flight Ctr. (United States); E. W. Dunham, Lowell Observatory (United States); J. C. Geary, Smithsonian Astrophysical Observatory (United States); P. Tenenbaum, NASA Ames Research Ctr. (United States); H. Chandrasekaran, NASA Ames Research Ctr. (United States) and Lawrence Livermore National Lab. (United States); J. Li, H. WU, NASA Ames Research Ctr. (United States); J. Von Wilpert, Univ. of California, Santa Cruz (United States)

773118 ASPIICS: a giant coronagraph for the ESA/PROBA-3 Formation Flying Mission [7731-44] P. Lamy, Lab. d'Astrophysique de Marseille, CNRS, Univ. de Provence (France); L. Damé, LATMOS/IPSL, CNRS, Univ. Versailles St-Quentin (France); S. Vivès, Lab. d'Astrophysique de Marseille, CNRS, Univ. de Provence (France); A. Zhukov, Royal Observatory of Belgium (Belgium)

773119 The narrow angle camera of the MPCS suite for the MarcoPolo ESA Mission: requirements and optical design solutions [7731-45]

V. Da Deppo, CNR-IFN UOS LUXOR Padova (Italy); G. Cremonese, INAF, Osservatorio Astronomico di Padova (Italy); G. Naletto, Univ. degli Studi di Padova (Italy) and CNR-IFN UOS LUXOR Padova (Italy)

7731 1A Stray light performance of the long range reconnaissance imager (LORRI) on the New Horizons Mission [7731-46]

A. F. Cheng, S. J. Conard, H. A. Weaver, F. Morgan, M. Noble, Johns Hopkins Univ. Applied Physics Lab. (United States)

7731 IB SPEX: the spectropolarimeter for planetary exploration [7731-47]

F. Snik, Sterrekundig Instituut, Utrecht Univ. (Netherlands); J. H. H. Rietjens, SRON Nationaal Instituut voor Ruimteonderzoek (Netherlands); G. van Harten, Sterrekundig Instituut, Utrecht Univ. (Netherlands); D. M. Stam, SRON Nationaal Instituut voor Ruimteonderzoek (Netherlands); C. U. Keller, Sterrekundig Instituut, Utrecht Univ. (Netherlands); J. M. Smit, SRON Nationaal Instituut voor Ruimteonderzoek (Netherlands); E. C. Laan, A. L. Verlaan, TNO (Netherlands); R. ter Horst, R. Navarro, NOVA-ASTRON (Netherlands); K. Wielinga, MECON Engineering B.V. (Netherlands); S. G. Moon, Cosine (Netherlands); R. Voors, Dutch Space B.V. (Netherlands) 
7731 1C Gaia: 1,000 million stars with 100 CCD detectors (Invited Paper) [7731-48]

J. de Bruijne, European Space Research and Technology Ctr. (Netherlands); R. Kohley, European Space Astronomy Ctr. (Spain); T. Prusti, European Space Research and Technology Ctr. (Netherlands)

\section{SESSION 14 JDEM}

7731 1D Joint Dark Energy Mission optical design studies [7731-49]

D. A. Content, NASA Goddard Space Flight Ctr. (United States); M. G. Dittman, B. Firth, Ball Aerospace \& Technologies Corp. (United States); J. M. Howard, C. E. Jackson, NASA Goddard Space Flight Ctr. (United States); J. P. Lehan, Univ. of Maryland, Baltimore County (United States); J. E. Mentzell, B. A. Pasquale, NASA Goddard Space Flight Ctr. (United States); M. J. Sholl, Lawrence Berkeley National Lab. (United States)

7731 1E Optical performance budgeting for JDEM weak-lensing measurements [7731-50] C. Noecker, Ball Aerospace \& Technologies Corp. (United States)

7731 IF Wide-field spectroscopy and imaging at two plate scales with a focal three mirror anastigmat [7731-51]

M. J. Sholl, Space Sciences Lab., Univ. of California, Berkeley (United States); D. A. Content, NASA Goddard Space Flight Ctr. (United States); M. L. Lampton, Space Sciences Lab., Univ. of California, Berkeley (United States); J. P. Lehan, Univ. of Maryland, Baltimore County (United States); M. E. Levi, Lawrence Berkeley National Lab. (United States)

7731 IG Off-axis telescopes for dark energy investigations [7731-52]

M. L. Lampton, M. J. Sholl, Space Sciences Lab., Univ. of California, Berkeley (United States); M. E. Levi, Lawrence Berkeley National Lab. (United States)

\section{SESSION 15 EUCLID}

7731 1H The Euclid Mission [7731-53]

R. J. Laureijs, L. Duvet, I. Escudero Sanz, P. Gondoin, D. H. Lumb, T. Oosterbroek, G. Saavedra Criado, European Space Research and Technology Ctr. (Netherlands)

$773111 \quad$ Euclid imaging channels: from science to system requirements [7731-54]

J. Amiaux, J. L. Auguères, O. Boulade, C. Cara, S. Paulin-Henriksson, A. Réfrégier, S. Ronayette, Service d'Astrophysique, CEA (France); A. Amara, ETH Zurich (Switzerland) A. Glauser, ETH Zurich (Switzerland) and UK Astronomy Technology Ctr. (United Kingdom); C. Dumesnil, Institut d'Astrophysique Spatiale, CNRS, Univ. Paris-Sud 11 (France); A. M. Di Giorgio, INAF, Istituto di Fisica dello Spazio Interplanetario (Italy); J. Booth, Jet Propulsion Lab. (United States); M. Schweitzer, Max-Planck-Institut für extraterrestrische Physik (Germany); R. Holmes, Max-Planck-Institut für Astronomie (Germany); M. Cropper, Mullard Space Science Lab., Univ. College London (United Kingdom); E. Atad-Ettedgui, UK Astronomy Technology Ctr. (United Kingdom); L. Duvet, D. Lumb, European Space Research and Technology Ctr. (Netherlands) 
7731 1J VIS: the visible imager for Euclid [7731-55]

M. Cropper, Mullard Space Science Lab., Univ. College London (United Kingdom); A. Refregier, Service d'Astrophysique, CEA (France); P. Guttridge, Mullard Space Science Lab., Univ. College London (United Kingdom); O. Boulade, J. Amiaux, Service d'Astrophysique, CEA (France); D. Walton, P. Thomas, K. Rees, Mullard Space Science Lab. Univ. College London (United Kingdom); P. Pool, J. Endicott, e2v technologies plc (United Kingdom); A. Holland, J. Gow, N. Murray, Planetary and Space Sciences Research Institute, The Open Univ. (United Kingdom); A. Amara, ETH Zurich (Switzerland); D. Lumb, L. Duvet, European Space Research and Technology Ctr. (Netherlands); R. Cole, Mullard Space Science Lab., Univ. College London (United Kingdom); J.-L. Augueres, Service d'Astrophysique, CEA (France); G. Hopkinson, Surrey Satellite Technology Ltd. (United Kingdom)

7731 1K NIP: the near infrared imaging photometer for Euclid [7731-56]

M. Schweitzer, R. Bender, R. Katterloher, F. Eisenhaver, R. Hofmann, R. Saglia, Max-Planck-Institut für extraterrestrische Physik (Germany); R. Holmes, O. Krause, H.-W. Rix, Max-Planck-Institut für Astronomie (Germany); J. Booth, P. Fagrelius, J. Rhodes, S. Seshadri, Jet Propulsion Lab. (United States); A. Refregier, J. Amiaux, J.-L. Augueres, O. Boulade, C. Cara, Service d'Astrophysique, CEA (France); A. Amara, S. Lilly, ETH Zurich (Switzerland); E. Atad-Ettedgui, The Royal Observatory, Edinburgh (United Kingdom); A.-M. Di Giorgio, INAF, Istituto di Fisica dello Spazio Interplanetario (Italy); L. Duvet, European Space Research and Technology Ctr. (Netherlands); C. Kuehl, M. Syed, EADS Astrium GmbH (Germany)

7731 1L The E-NIS instrument on-board the ESA Euclid Dark Energy Mission: a general view after positive conclusion of the assessment phase [7731-58]

L. Valenziano, INAF, Istituto Astrofisica Spaziale Fisica Cosmica (Italy); F. M. Zerbi, INAF, Osservatorio Astronomico di Brera (Italy); A. Cimatti, Univ. degli Studi di Bologna (Italy); A. Bianco, INAF, Osservatorio Astronomico di Brera (Italy); C. Bonoli, F. Bortoletto, INAF, Osservatorio Astronomico di Padova (Italy); A. Bulgarelli, R. C. Butler, INAF, Istituto Astrofisica Spaziale Fisica Cosmica (Italy); R. Content, Durham Univ. (United Kingdom); L. Corcione, INAF, Osservatorio Astronomico di Torino (Italy); A. De Rosa, INAF, Istituto Astrofisica Spaziale Fisica Cosmica (Italy); P. Franzetti, B. Garilli, INAF, Istituto di Astrofisica Spaziale di Milano (Italy); F. Gianotti, INAF, Istituto Astrofisica Spaziale Fisica Cosmica (Italy); E. Giro, INAF, Osservatorio Astronomico di Padova (Italy); R. Grange, Lab. d'Astrophysique de Marseille, CNRS (France); P. Leutenegger, Thales Alenia Space (Italy); S. Ligori, INAF, Osservatorio Astronomico di Padova (Italy); L. Martin, Lab. d'Astrophysique de Marseille, CNRS (France); N. Mandolesi, G. Morgante, L. Nicastro, INAF, Istituto Astrofisica Spaziale Fisica Cosmica (Italy); M. Riva, INAF, Osservatorio Astronomico di Brera (Italy); M. Robberto, Space Telescope Science Institute (United States); R. Sharples, Durham Univ. (United Kingdom); P. Spanò, INAF, Osservatorio Astronomico di Brera (Italy); G. Talbot, Durham Univ. (United Kingdom); M. Trifoglio, INAF, Istituto Astrofisica Spaziale Fisica Cosmica (Italy); R. Wink, TNO Science and Industry (Netherlands); F. Zamkotsian, Lab. d'Astrophysique de Marseille, CNRS (France)

\section{SESSION 16 SYSTEMS CONCEPTS I}

7731 1M Definition phase activities for ESA's Cosmic Vision mission PLATO [7731-59]

A. Stankov, M. Baldesarra, O. Piersanti, M. Fridlund, R. Lindberg, N. Rando, European Space Research and Technology Ctr. (Netherlands) 
7731 iN A 4-meter wide field coronagraph space telescope for general astrophysics and exoplanet observations [7731-60]

D. Tenerelli, Lockheed Martin Space Systems Co. (United States); R. Angel, J. Burge, The Univ. of Arizona (United States); O. Guyon, The Univ. of Arizona (United States) and National Astronomical Observatory of Japan/Subaru Telescope (United States); A. Zabludoff, The Univ. of Arizona (United States); R. Belikov, E. Pluzhnik, ARC-SSA (United States); R. Egerman, ITT Corp. (United States)

773110 A space imaging concept based on a $4 \mathrm{~m}$ structured spun-cast borosilicate monolithic primary mirror [7731-61]

S. C. West, S. H. Bailey, Steward Observatory, The Univ. of Arizona (United States); S. Bauman, Canada-France-Hawaii Telescope (United States); B. Cuerden, Steward Observatory, The Univ. of Arizona (United States); Z. Granger, Lockheed Martin Space Systems Co. (United States); B. H. Olbert, Steward Observatory, The Univ. of Arizona (United States)

7731 iP A general purpose astronomy small satellite: an approach to low-cost space telescope design using space-qualified ground telescopes [7731-62]

N. Bosanac, S. Do, H. Y. Wen, A. Wicht, Massachusetts Institute of Technology (United States)

$77311 Q \quad$ WISH: wide-field imaging surveyor at high redshift [7731-63]

T. Yamada, Astronomical Institute, Tohoku Univ. (Japan); M. Doi, Institute of Astronomy, The Univ. of Tokyo (Japan); T. Goto, Institute for Astronomy, Univ. of Hawai'i (United States); Y. Ikeda, Photocoding (Japan); M. Imanishi, National Astronomical Observatory of Japan (Japan); A. Inove, Osaka Sangyo Univ. (Japan); S. Iwamura, Montgomery, Rennie \& Jonson, LPA (Japan); I. Iwata, Okayama Astrophysical Observatory, National Astronomical Observatory of Japan (Japan); N. Kawai, Tokyo Institute of Technology (Japan); M. A. R. Kobayashi, T. Kodama, Y. Komiyama, National Astronomical Observatory of Japan (Japan); H. Matsuhara, Japan Aerospace Exploration Agency (Japan); Y. Matsuoka, Nagoya Univ. (Japan); T. Morokuma, National Astronomical Observatory of Japan (Japan) and Japan Society for the Promotion of Science (Japan); K. Ohta, Kyoto Univ. (Japan); S. Oyabu, Subaru Telescope, National Astronomical Observatory of Japan (United States); Y. Sato, H. Sugita, Japan Aerospace Exploration Agency (Japan); R. Tsutsui, Kyoto Univ. (Japan); C. Tokoku, Astronomical Institute, Tohoku Univ. (Japan); S. Tsuneta, National Astronomical Observatory of Japan (Japan); T. Wada, Japan Aerospace Exploration Agency (Japan); K. Yabe, Kyoto Univ. (Japan); N. Yasuda, Institute for the Physics and Mathematics of the Universe, The Univ. of Tokyo (Japan); D. Yonetoku, Kanazawa Univ. (Japan)

\section{SESSION 17 SYSTEMS CONCEPTS II}

7731 IR Optical design of the EPIC-IM crossed Dragone telescope [7731-64]

H. Tran, B. Johnson, Univ. of California, Berkeley (United States); M. Dragovan, Jet Propulsion Lab. (United States); J. Bock, Jet Propulsion Lab. (United States) and California Institute of Technology (United States); A. Aljabri, Jet Propulsion Lab. (United States); A. Amblard, Univ. of California, Irvine (United States); D. Bauman, Harvard Univ. (United States); M. Betoule, Institut d'Astrophysique Spatiale (France); T. Chui, Jet Propulsion Lab. (United States); L. Colombo, The Univ. of Southern California (United States); A. Cooray, Univ. of California, Irvine (United States); D. Crumb, ATK Aerospace Systems (United States); P. Day, Jet Propulsion Lab. (United States); C. Dickenson, California Institute of Technology (United States) and Jodrell Bank Ctr. for Astrophysics, The Univ. of Manchester (United Kingdom); D. Dowell, Jet Propulsion Lab. (United States) and California Institute of Technology (United 
States); S. Golwala, California Institute of Technology (United States); K. Gorski, Jet Propulsion Lab. (United States) and California Institute of Technology (United States); S. Hanany, Univ. of Minnesota, Minneapolis (United States); W. Holmes, Jet Propulsion Lab. (United States); K. Irwin, National Institute of Standards and Technology (United States); B. Keating, Univ. of California, San Diego (United States); C.-L. Kuo, Stanford Univ. (United States); A. Lee, Univ. of California, Berkeley (United States); A. Lange, California Institute of Technology (United States) and Jet Propulsion Lab. (United States); C. Lawrence, Jet Propulsion Lab. (United States); S. Meyer, The Univ. of Chicago (United States); N. Miller, Univ. of California, San Diego (United States); H. Nguyen, Jet Propulsion Lab. (United States); E. Pierpaoli, The Univ. of Southern California (United States); N. Ponthieu, J.-L. Puget, Institut d'Astrophysique Spatiale (France); J. Raab, Northrop Grumman Aerospace Systems (United States);

P. Richards, Univ. of California, Berkeley (United States); C. Satter, M. Seiffert, Jet Propulsion Lab. (United States); M. Shimon, Univ. of California, San Diego (United States); B. Williams, Jet Propulsion Lab. (United States); J. Zmuidzinas, California Institute of Technology (United States) and Jet Propulsion Lab. (United States)

7731 is The Primordial Inflation Explorer (PIXIE) Mission [7731-65]

A. J. Kogut, D. T. Chuss, NASA Goddard Space Flight Ctr. (United States); J. L. Dotson, NASA Ames Research Ctr. (United States); D. J. Fixsen, NASA Goddard Space Flight Ctr. (United States); M. Halpern, The Univ. of British Columbia (Canada); G. F. Hinshaw, NASA Goddard Space Flight Ctr. (United States); S. M. Meyer, The Univ. of Chicago (United States);

S. H. Moseley, NASA Goddard Space Flight Ctr. (United States); M. D. Seiffert, The Univ. of Chicago (United States); D. N. Spergel, Princeton Univ. (United States); E. J. Wollack, NASA Goddard Space Flight Ctr. (United States)

7731 IT LEGOLAS: localizing evidence of gravitational waves by observations of light source astrometric signature [7731-66]

A. Riva, D. Busonero, M. Gai, M. T. Crosta, A. Vecchiato, M. G. Lattanzi, INAF, Osservatorio Astronomico di Torino (Italy)

$77311 \mathrm{U}$ Design of a four mirror astrometric telescope for light bending measurements [7731-67] D. Loreggia, M. Gai, S. Ligori, A. Vecchiato, M. G. Lattanzi, INAF, Osservatorio Astronomico di Torino (Italy)

7731 IV Achieving high-precision pointing on ExoplanetSat: initial feasibility analysis [7731-68] C. M. Pong, Massachusetts Institute of Technology (United States); S. Lim, The Charles Stark Draper Lab., Inc. (United States); M. W. Smith, D. W. Miller, J. S. Villaseñor, S. Seager, Massachusetts Institute of Technology (United States)

\section{Part Two}

\section{SESSION 18 SYSTEMS CONCEPTS III}

7731 IW System design of the compact IR space imaging system MIRIS [7731-69]

W. Han, D.-H. Lee, Y. Park, W.-S. Jeong, C. H. Ree, B. Moon, S.-M. Cha, S.-J. Park, J.-H. Park, U.-W. Nam, N. H. Ka, M. H. Lee, J. Pyo, K.-I. Seon, Korea Astronomy and Space Science Institute (Korea, Republic of); D.-H. Lee, Korea Astronomy and Space Science Institute (Korea, Republic of) and Univ. of Science and Technology (Korea, Republic of); S. C. Yang, Korea Basic Science Institute (Korea, Republic of); S.-W. Rhee, J.-O. Park, Korea Aerospace Research Institute (Korea, Republic of); H. M. Lee, Seoul National Univ. (Korea, Republic of); 
T. Matsumoto, Seoul National Univ. (Korea, Republic of) and Institute of Space and Astronautical Science (Japan)

7731 1X Optical design and performance of MIRIS near-infrared camera [7731-166]

C. H. Ree, S.-J. Park, B. Moon, S.-M. Cha, Y. Park, W.-S. Jeong, D.-H. Lee, U.-W. Nam, J.-H. Park, N. H. Ka, M. H. Lee, J. Pyo, Korea Astronomy and Space Science Institute (Korea, Republic of); D.-H. Lee, Korea Astronomy and Space Science Institute (Korea, Republic of) and Univ. of Science and Technology (Korea, Republic of); S.-W. Rhee, J.-O. Park, Korea Aerospace Research Institute (Korea, Republic of); H. M. Lee, Seoul National Univ. (Korea, Republic of); T. Matsumoto, Seoul National Univ. (Korea, Republic of) and Institute of Space and Astronautical Science (Japan); S. C. Yang, Korea Basic Science Institute (Korea, Republic of); W. Han, Korea Astronomy and Space Science Institute (Korea, Republic of)

7731 IY Development of mechanical structure for the compact space IR camera MIRIS [7731-167] B. Moon, W.-S. Jeong, S.-M. Cha, Y. Park, C. H. Ree, D.-H. Lee, S.-J. Park, U.-W. Nam, J.-H. Park, N. H. Ka, M. H. Lee, Korea Astronomy and Space Science Institute (Korea, Republic of); D.-H. Lee, Korea Astronomy and Space Science Institute (Korea, Republic of) and Univ. of Science and Technology (Korea, Republic of); J. Pyo, Korea Astronomy and Space Science Institute (Korea, Republic of); S.-W. Rhee, J.-O. Park, Korea Aerospace Research Institute (Korea, Republic of); H. M. Lee, Seoul National Univ. (Korea, Republic of); T. Matsumoto, Seoul National Univ. (Korea, Republic of) and Institute of Space and Astronautical Science (Japan); S. C. Yang, Korea Basic Science Institute (Korea, Republic of); W. Han, Korea Astronomy and Space Science Institute (Korea, Republic of)

773112 The design and capabilities of the EXIST optical and infra-red telescope (IRT) [7731-70] A. S. Kutyrev, Univ. of Maryland, College Park (United States) and NASA Goddard Space Flight Ctr. (United States); S. H. Moseley, NASA Goddard Space Flight Ctr. (United States); C. Golisano, ITT Corp. (United States); Q. Gong, NASA Goddard Space Flight Ctr. (United States); B. T. Allen, Harvard-Smithsonian Ctr. for Astrophysics (United States); N. Gehrels, NASA Goddard Space Flight Ctr. (United States); J. E. Grindlay, J. S. Hong, HarvardSmithsonian Ctr. for Astrophysics (United States); B. E. Woodgate, NASA Goddard Space Flight Ctr. (United States)

773120 Actuated hybrid mirrors for space telescopes [7731-71] G. Hickey, Jet Propulsion Lab. (United States); T. Barbee, Lawrence Livermore National Lab. (United States); M. Ealey, Northrop Grumman Xinetics (United States); D. Redding, Jet Propulsion Lab. (United States)

773121 Shape correction of thin mirrors in a reconfigurable modular space telescope [7731-72] K. Patterson, S. Pellegrino, J. Breckinridge, Graduate Aerospace Labs., California Institute of Technology (United States)

773122 Minimizing actuator-induced errors in active space telescope mirrors [7731-73] M. W. Smith, D. W. Miller, Massachusetts Institute of Technology (United States)

773123 Membrane photon sieve telescopes [7731-74]

G. Andersen, Air Force Academy (United States) 
773124 PLATO: detailed design of the telescope optical units [7731-75]

D. Magrin, INAF, Osservatorio Astronomico di Padova (Italy); M. Munari, I. Pagano, INAF, Osservatorio Astrofisico di Catania (Italy); D. Piazza, Univ. Bern (Switzerland); R. Ragazzoni, INAF, Osservatorio Astronomico di Padova (Italy); C. Arcidiacono, INAF, Osservatorio Astronomico di Arcetri (Italy); S. Basso, INAF, Osservatorio Astronomico di Brera (Italy); M. Dima, J. Farinato, INAF, Osservatorio Astronomico di Padova (Italy); L. Gambicorti, Istituto Nazionale di Ottica Applicata (Italy); G. Gentile, INAF, Osservatorio Astronomico di Padova (Italy); M. Ghigo, INAF, Osservatorio Astronomico di Brera (Italy); E. Pace, Univ. degli Studi di Firenze (Italy); G. Piotto, Univ. degli Studi di Padova (Italy); S. Scuderi, INAF, Osservatorio Astrofisico di Catania (Italy); V. Viotto, INAF, Osservatorio Astronomico di Padova (Italy); W. Zima, Instituut voor Sterrenkunde, Katholieke Univ. Leuven (Belgium); C. Catala, Observatoire de Paris à Meudon (France)

773125 THESIS: the terrestrial habitable-zone exoplanet spectroscopy infrared spacecraft [7731-76] M. R. Swain, G. Vasisht, Jet Propulsion Lab. (United States); T. Henning, Max-Planck Institut für Astronomie (Germany); G. Tinetti, Univ. College London (United Kingdom); J.-P. Beaulieu, Institut d'Astrophysique de Paris (France)

773126 See-coast: polarimetric and spectral characterization of exoplanets with a small space telescope [7731-77]

R. Galicher, LUTH, CNRS, Observatoire de Paris à Meudon (France) and Partenariat Haute Résolution Angulaire Sol Espace (France); P. Baudoz, A. Boccaletti, A.-L. Maire, LESIA, CNRS, Observatoire de Paris à Meudon (France) and Partenariat Haute Résolution Angulaire Sol Espace (France); S. Ronayette, CEA Saclay (France); J. Schneider, LUTH, CNRS, Observatoire de Paris à Meudon (France)

773127 ExoplanetSat: detecting transiting exoplanets using a low-cost CubeSat platform [7731-78] M. W. Smith, S. Seager, C. M. Pong, Massachusetts Institute of Technology (United States); J. S. Villaseñor, G. R. Ricker, Kavli Institute for Astrophysics and Space Research, Massachusetts Institute of Technology (United States); D. W. Miller, M. E. Knapp, G. T. Farmer, R. Jensen-Clem, Massachusetts Institute of Technology (United States)

\section{SESSION 20 TPF CORONAGRAPH}

773128 ACCESS: a concept study for the direct imaging and spectroscopy of exoplanetary systems [7731-79]

J. Trauger, K. Stapelfeldt, W. Traub, J. Krist, D. Moody, D. Mawet, E. Serabyn, C. Henry, P. Brugarolas, J. Alexander, R. Gappinger, O. Dawson, V. Mireles, P. Park, L. Pueyo, S. Shaklan, Jet Propulsion Lab. (United States); O. Guyon, Subaru Telescope, National Astronomical Observatory of Japan (United States); and The Univ. of Arizona (United States); J. Kasdin, R. Vanderbei, D. Spergel, Princeton Univ. (United States); R. Belikov, NASA Ames Research Ctr. (United States); G. Marcy, Univ. of California, Berkeley (United States); R. A. Brown, Space Telescope Science Institute (United States); J. Schneider, Observatoire de Paris à Meudon (France); B. Woodgate, NASA Goddard Space Flight Ctr. (United States); R. Egerman, G. Matthews, J. Elias, Y. Conturie, P. Vallone, P. Voyer, ITT Corp. (United States); R. Polidan, C. Lillie, C. Spittler, D. Lee, R. Hejal, A. Bronowicki, N. Saldivar, Northrop Grumman Corp. (United States); M. Ealey, T. Price, Northrop Grumman Xinetics (United States) 
773129 The pupil mapping exoplanet coronagraphic observer (PECO) [7731-80]

O. Guyon, Steward Observatory, The Univ. of Arizona (United States); S. Shaklan, M. Levine, Jet Propulsion Lab. (United States); K. Cahoy, NASA Ames Research Ctr. (United States);

D. Tenerelli, Lockheed Martin Corp. (United States); R. Belikov, NASA Ames Research Ctr. (United States); B. Kern, Jet Propulsion Lab. (United States)

7731 2A Optical design of dilute aperture visible nulling coronagraph imaging (DAViNCI) [7731-81] R. A. Woodruff, Lockheed Martin Space Systems Co. (United States); M. Shao, B. M. Levine, Jet Propulsion Lab. (United States); R. Lyon, NASA Goddard Space Flight Ctr. (United States); G. Vasudevan, J. ACU, Lockheed Martin Advanced Technology Ctr. (United States); K. Havey, J. Wynn, ITT Geospatial Systems (United States)

7731 2B Visible nulling coronagraphy testbed development for exoplanet detection [7731-82] R. G. Lyon, M. Clampin, NASA Goddard Space Flight Ctr. (United States); R. A. Woodruff, Lockheed Martin Space Systems Co. (United States); G. Vasudevan, Lockheed Martin Advanced Technology Ctr. (United States); P. Thompson, NASA Goddard Space Flight Ctr. (United States); A. Chen, Lockheed Martin Corp. (United States); P. Petrone, A. Booth, Sigma Space Corp. (United States); T. Madison, M. Bolcar, NASA Goddard Space Flight Ctr. (United States); M. C. Noecker, S. Kendrick, Ball Aerospace \& Technology Corp. (United States); G. Melnick, V. Tolls, Harvard-Smithsonian Ctr. for Astrophysics (United States)

7731 2C Single aperture imaging astrometry with a diffracting pupil: application to exoplanet mass measurement with a small coronagraphic space telescope [7731-83]

O. Guyon, Steward Observatory, The Univ. of Arizona (United States); M. Shao, S. Shaklan, M. Levine, Jet Propulsion Lab. (United States); M. Ammons, E. Bendek, Steward Observatory, The Univ. of Arizona (United States); R. Woodruff, Lockheed Martin Space Systems Co. (United States); B. Nemati, Jet Propulsion Lab. (United States); J. Pitman, Exploration Sciences (United States)

$77312 \mathrm{D} \quad$ Laboratory demonstration of high-contrast imaging at $2 \lambda / D$ on a temperature-stabilized testbed in air [7731-84]

R. Belikov, E. Pluzhnik, M. S. Connelley, F. C. Witteborn, T. P. Greene, D. H. Lynch, P. T. Zell, NASA Ames Research Ctr. (United States); O. Guyon, The Univ. of Arizona (United States)

\section{SESSION $21 \quad$ TPF OCCULTER}

$77312 \mathrm{E} \quad$ New Worlds Probe [7731-85]

A. S. Lo, T. Glassman, D. Dailey, K. Sterk, J. Green, Northrop Grumman Aerospace Systems (United States); W. Cash, Univ. of Colorado at Boulder (United States); R. Soummer, Space Telescope Science Institute (United States)

$77312 \mathrm{~F} \quad$ Broadband suppression and occulter position sensing at the Princeton occulter testbed [7731-86]

E. Cady, Princeton Univ. (United States) and Jet Propulsion Lab. (United States);

K. Balasubramanian, Jet Propulsion Lab. (United States); M. Carr, Princeton Univ. (United States); M. Dickie, P. Echternach, Jet Propulsion Lab. (United States); J. Kasdin, Princeton Univ. (United States); S. Shaklan, Jet Propulsion Lab. (United States); D. Sirbu, Princeton Univ. (United States); V. White, Jet Propulsion Lab. (United States) 
77312 E Error budgeting and tolerancing of starshades for exoplanet detection (Invited Paper) [7731-87]

S. B. Shaklan, Jet Propulsion Lab. (United States); M. C. Noecker, Ball Aerospace \& Technologies Corp. (United States); T. Glassman, A. S. Lo, Northrop Grumman Aerospace Systems (United States); P. J. Dumont, Jet Propulsion Lab. (United States); N. J. Kasdin, E. J. Cady, R. Vanderbei, Princeton Univ. (United States); P. R. Lawson, Jet Propulsion Lab. (United States)

$77312 \mathrm{H} \quad$ Occulting ozone observatory science overview [7731-88]

D. Savransky, D. N. Spergel, N. J. Kasdin, E. J. Cady, Princeton Univ. (United States); P. D. Lisman, S. H. Pravdo, S. B. Shaklan, Jet Propulsion Lab. (United States) and California Institute of Technology (United States); Y. Fujii, The Univ. of Tokyo (Japan)

$773121 \quad$ Direct imaging and spectroscopy of habitable planets using JWST and a starshade [7731-89] R. Soummer, J. Valenti, R. A. Brown, Space Telescope Science Institute (United States); S. Seager, Massachusetts Institute of Technology (United States); J. Tumlinson, Space Telescope Science Institute (United States); W. Cash, Univ. of Colorado at Boulder (United States); I. Jordan, Computer Sciences Corp. (United States) and Space Telescope Science Institute (United States); M. Postman, M. Mountain, Space Telescope Science Institute (United States); T. Glassman, Northrop Grumman Aerospace Systems (United States); L. Pueyo, Jet Propulsion Lab. (United States); A. Roberge, NASA Goddard Space Flight Ctr. (United States)

7731 2J Alternative starshade missions [7731-90] W. Cash, Univ. of Colorado at Boulder (United States); T. Glassman, A. Lo, Northrop Grumman Aerospace Systems (United States); R. Soummer, Space Telescope Science Institute (United States)

\section{SESSION 22 ATLAST}

7731 2K Science drivers and requirements for an Advanced Technology Large Aperture Space Telescope (ATLAST): implications for technology development and synergies with other future facilities [7731-91]

M. Postman, T. Brown, K. Sembach, Space Telescope Science Institute (United States); M. Giavalisco, Univ. of Massachusetts Amherst (United States); W. Traub, K. Stapelfeldt, Jet Propulsion Lab. (United States); D. Calzetti, Univ. of Massachusetts Amherst (United States); W. Oegerle, NASA Goddard Space Flight Ctr. (United States); R. M. Rich, Univ. of California, Los Angeles (United States); H. P. Stahl, NASA Marshall Space Flight Ctr. (United States); J. Tumlinson, M. Mountain, R. Soummer, Space Telescope Science Institute (United States); T. Hyde, NASA Goddard Space Flight Ctr. (United States)

7731 2L Comparative concepts for ATLAST optical designs [7731-92] B. A. Pasquale, NASA Goddard Space Flight Ctr. (United States); P. Stahl, NASA Marshall Space Flight Ctr. (United States); L. Feinberg, J. Howard, Q. Gong, D. Aronstein, NASA Goddard Space Flight Ctr. (United States) 
$77312 \mathrm{M}$ ATLAST-9.2m: a large-aperture deployable space telescope [7731-93]

W. R. Oegerle, L. D. Feinberg, L. R. Purves, T. T. Hyde, H. A. Thronson, J. A. Townsend, NASA Goddard Space Flight Ctr. (United States); M. Postman, Space Telescope Science Institute (United States); M. R. Bolcar, J. G. Budinoff, B. H. Dean, M. C. Clampin, NASA Goddard Space Flight Ctr. (United States); D. C. Ebbets, Ball Aerospace \& Technologies Corp. (United States); Q. Gong, T. R. Gull, J. M. Howard, A. L. Jones, R. G. Lyon, B. A. Pasquale, C. Perrygo, J. S. Smith, P. L. Thompson, B. E. Woodgate, NASA Goddard Space Flight Ctr. (United States)

7731 2N ATLAST-8 Mission concept study for 8-meter monolithic UV/optical space telescope [7731-94]

H. P. Stahl, NASA Marshall Space Flight Ctr. (United States); M. Postman, Space Telescope Science Institute (United States); W. R. Arnold, Sr., R. C. Hopkins, L. Hornsby, NASA Marshall Space Flight Ctr. (United States); G. E. Mosier, B. A. Pasquale, NASA Goddard Space Flight Ctr. (United States)

\section{SESSION 23 LATE BREAKING NEWS}

773120 The potential of small space telescopes for exoplanet observations (Invited Paper) [7731-197]

E. Serabyn, D. Mawet, R. Burruss, Jet Propulsion Lab. (United States)

\section{POSTER SESSION: AKARI}

7731 2P AKARI infrared bright source catalogues [7731-95]

S. Oyabu, Japan Aerospace Exploration Agency (Japan) and Subaru Telescope, National Astronomical Observatory of Japan (Japan); I. Yamamura, Japan Aerospace Exploration Agency (Japan); C. Alfageme, European Space Astronomy Ctr. (Spain); P. Barthel, Kapteyn Astronomical Institute, Univ. of Groningen (Netherlands); A. Cassatella, European Space Astronomy Ctr. (Spain); M. Cohen, Radio Astronomy Lab., Univ. of California, Berkeley (United States); N. Cox, European Space Astronomy Ctr. (Spain); E. Figueredo, The Open Univ. (United Kingdom) and Univ. of Sao Paulo (Brazil); H. Fujiwara, N. Ikeda, Japan Aerospace Exploration Agency (Japan); D. Ishihara, Nagoya Univ. (Japan); W.-S. Jeong, Korea Astronomy and Space Science Institute (Korea, Republic of); H. Kataza, Japan Aerospace Exploration Agency (Japan); D. Kester, SRON National Institute for Space Research (Netherlands); H. M. Lee, Seoul National Univ. (Korea, Republic of); S. Makiuti, Japan Aerospace Exploration Agency (Japan); T. G. Mueller, Max-Planck-Institut für extraterrestrische Physik (Germany); T. Nakagawa, S. Takita, Japan Aerospace Exploration Agency (Japan); S. H. Oh, Seoul National Univ. (Korea, Republic of) and National Institute of Mathematical Sciences (Korea, Republic of); S. Oliver, Astronomy Ctr., Univ. of Sussex (United Kingdom); C. Pearson, Rutherford Appleton Lab. (United Kingdom) and Univ. of Lethbridge (Canada); N. Rahman, Astronomy Ctr., Univ. of Sussex (United Kingdom) and Univ. of Maryland, College Park (United States); M. Rowan-Robinson, Imperial College of Science Technology and Medicine (United Kingdom); A. Salama, European Space Astronomy Ctr. (Spain); R. Savage, Astronomy Ctr., Univ. of Sussex (United Kingdom) and Univ. of Warwick (United Kingdom); S. Serjeant, The Open Univ. (United Kingdom); G. J. White, The Open Univ. (United Kingdom) and Rutherford Appleton Lab. (United Kingdom); C. Yamauchi, Japan Aerospace Exploration Agency (Japan) 
$77312 \mathrm{Q}$ Spacecraft conceptual design for the 8-meter Advanced Technology Large Aperture Space Telescope (ATLAST) [7731-96]

R. C. Hopkins, NASA Marshall Space Flight Ctr. (United States); P. Capizzo, Raytheon, NASA Marshall Space Flight Ctr. (United States); S. Fincher, L. S. Hornsby, D. Jones, NASA Marshall Space Flight Ctr. (United States); G. Mosier, NASA Goddard Space Flight Ctr. (United States); H. P. Stahl, D. Thomas, K. S. Thompson, NASA Marshall Space Flight Ctr. (United States)

$77312 R \quad$ Thermal analysis of the Advanced Technology Large Aperture Space Telescope (ATLAST) 8-meter primary mirror [7731-97]

L. Hornsby, R. C. Hopkins, H. P. Stahl, NASA Marshall Space Flight Ctr. (United States)

$77312 S \quad$ Coronagraphic wavefront control for the ATLAST 9.2m telescope [7731-98]

R. G. Lyon, W. R. Oegerle, L. D. Feinberg, M. R. Bolcar, B. H. Dean, G. E. Mosier, NASA Goddard Space Flight Ctr. (United States); M. Postman, Space Telescope Science Institute (United States)

\section{POSTER SESSION: EUCLID}

$77312 T \quad$ Euclid ENIS spectrograph focal-plane design [7731-99] F. Bortoletto, C. Bonoli, M. D'Alessandro, E. Giro, INAF, Osservatorio Astronomico di Padova (Italy); V. De Caprio, INAF-IASF Milano (Italy); L. Corcione, S. Ligori, INAF, Osservatorio Astronomico di Torino (Italy); G. Morgante, INAF-IASF Bologna (Italy)

$77312 \mathrm{U}$ A frame simulator for data produced by multi-accumulation readout detectors [7731-100] C. Bonoli, F. Bortoletto, E. Giro, INAF, Osservatorio Astronomico di Padova (Italy); L. Corcione, S. Ligori, INAF, Osservatorio Astronomico di Torino (Italy); L. Nicastro, INAF-IASF Bologna (Italy)

$77312 \mathrm{~V}$ The Euclid near-infrared calibration source [7731-101] R. Holmes, P. Bizenberger, O. Krause, Max-Planck-Institut für Astronomie (Germany); M. Schweitzer, Max-Planck-Institut für extraterrestrische Physik (Germany); A. M. Glauser, ETH Zurich (Switzerland) and UK Astronomy Technology Ctr. (United Kingdom)

7731 2W The data handling unit of the Euclid imaging channels: from the observational requirements to the unit architecture [7731-102]

A. M. Di Giorgio, INAF, Istituto di Fisica dello Spazio Interplanetario (Italy); P. H. Leutenegger, A. Bonati, Thales Alenia Space Italia S.p.A. (Italy); R. Scaramella, Osservatorio Astronomico di Roma (Italy); A. Refregier, J. Amiaux, C. Cara, J.-L. Augueres, Service d'Astrophysique, CEA (France); M. Schweitzer, Max-Planck-Institut für extraterrestrische Physik (Germany)

$77312 X$ The ground support equipment for the E-NIS instrument on-board the ESA-Euclid Dark Energy Mission in the baseline configuration presented in phase A [7731-103] M. Trifoglio, F. Gianotti, A. Bulgarelli, E. Franceschi, L. Nicastro, L. Valenziano, INAF, Istituto Astrofisica Spaziale Fisica Cosmica (Italy); F. M. Zerbi, INAF, Osservatorio Astronomico di Brera (Italy); A. Cimatti, Univ. degli Studi di Bologna (Italy)

$77312 Y \quad$ EUCLID: design of the prism DMD NIR spectrograph [7731-104]

R. Content, R. M. Sharples, S. Blake, R. G. Talbot, Durham Univ. (United Kingdom) 
$77312 Z$ Opto-mechanical design of a DMD multislit spectrograph for the ESA Euclid Mission [7731-105]

R. Grange, F. Zamkotsian, L. Martin, T. Pamplona, O. Le Fevre, Lab. d'Astrophysique de Marseille (France); L. Valenziano, INAF, Istituto Astrofisica Spaziale Fisica Cosmica (Italy); F. M. Zerbi, INAF, Osservatorio Astronomico di Brera (Italy); A. Cimatti, Osservatorio Astrofisico di Arcetri (Italy)

773130 Space evaluation of 2048×1080 mirrors DMD chip for ESA's EUCLID Mission [7731-106] F. Zamkotsian, P. Lanzoni, E. Grassi, R. Barette, C. Fabron, Lab. d'Astrophysique de Marseille, CNRS (France); K. Tangen, Visitech AS (Norway); L. Valenziano, INAF, Istituto Astrofisica Spaziale Fisica Cosmica (Italy); L. Marchand, L. Duvet, European Space Agency (Netherlands)

\section{POSTER SESSION: GAIA}

773131 Astrometric instrument model software tool for Gaia data reduction: challenges and implementation [7731-107]

D. Busonero, F. Russo, D. Loreggia, A. Riva, D. Bonino, L. Corcione, M. Gai, M. G. Lattanzi, INAF, Osservatorio Astronomico di Torino (Italy)

773132 Towards a demonstrator for autonomous object detection on board Gaia [7731-108] S. Mignot, Observatoire de Paris (France)

773133 Monitoring, diagnostic, and calibration of the Gaia astrometric instrument response within the astrometric verification unit [7731-109]

D. Busonero, M. Gai, M. G. Lattanzi, INAF, Osservatorio Astronomico di Torino (Italy)

\section{POSTER SESSION: HERSCHEL}

773134 The data processing pipelines for the Herschel/SPIRE imaging Fourier transform spectrometer [7731-110]

T. R. Fulton, Blue Sky Spectroscopy Inc. (Canada); J.-P. Baluteau, Lab. d'Astrophysique de Marseille (France); G. Bendo, Imperial College London (United Kingdom); D. Benielli, Lab. d'Astrophysique de Marseille (France); R. Gastaud, Lab. AIM, CEA Saclay (France); M. Griffin, Cardiff Univ. (United Kingdom); S. Guest, Rutherford Appleton Lab. (United Kingdom); P. Imhof, Blue Sky Spectroscopy Inc. (Canada); T. L. Lim, Rutherford Appleton Lab. (United Kingdom); N. Lu, California Institute of Technology (United States); D. A. Naylor, Institute for Space Imaging Science, Univ. of Lethbridge (Canada); P. Panuzzo, Lab. AIM, CEA Saclay (France); E. Polehampton, Rutherford Appleton Lab. (United Kingdom) and Institute for Space Imaging Science, Univ. of Lethbridge (Canada); A. Schwartz, California Institute of Technology (United States); C. Surace, Lab. d'Astrophysique de Marseille (France); B. M. Swinyard, Rutherford Appleton Lab. (United Kingdom); K. Xu, California Institute of Technology (United States)

773135 In-flight characterisation of Herschel-SPIRE optical performances [7731-111] M. Ferlet, Rutherford Appleton Lab. (United Kingdom) 
773136 Status of the SPIRE photometer data processing pipelines during the early phases of the Herschel Mission [7731-112]

C. D. Dowell, Jet Propulsion Lab. (United States); M. Pohlen, Cardiff Univ. (United Kingdom) and Rutherford Appleton Lab. (United Kingdom); C. Pearson, Rutherford Appleton Lab. (United Kingdom) and Institute for Space Imaging Science, Univ. of Lethbridge (United Kingdom); M. Griffin, Cardiff Univ. (United Kingdom); T. Lim, Rutherford Appleton Lab. (United Kingdom); G. J. Bendo, Imperial College London (United Kingdom); D. Benielli, Lab. d'Astrophysique de Marseille, CNRS (France); J. J. Bock, Jet Propulsion Lab. (United States); P. Chanial, Service d'Astrophysique, CEA (France) and Imperial College London (United Kingdom); D. L. Clements, Imperial College London (United Kingdom); L. Conversi, European Space Astronomy Ctr. (Spain); M. Ferlet, Rutherford Appleton Lab. (United Kingdom); T. Fulton, Blue Sky Spectroscopy Inc. (Canada); R. Gastaud, Service d'Astrophysique, CEA (France); J. Glenn, Univ. of Colorado at Boulder (United States); T. Grundy, S. Guest, K. J. King, S. J. Leeks, Rutherford Appleton Lab. (United Kingdom); L. Levenson, California Institute of Technology (United States); N. Lu, NASA Herschel Science Ctr. (United States); H. Morris, Rutherford Appleton Lab. (United Kingdom); H. Nguyen, Jet Propulsion Lab. (United States); B. O'Halloran, Imperial College London (United Kingdom); S. Oliver, Astronomy Ctr., Univ. of Sussex (United Kingdom); P. Panuzzo, Service d'Astrophysique, CEA (France); A. Papageorgiou, Cardiff Univ. (United Kingdom); E. Polehampton, Rutherford Appleton Lab. (United Kingdom) and Institute for Space Imaging Science, Univ. of Lethbridge (United Kingdom); D. Rigopoulou, Rutherford Appleton Lab. (United Kingdom); H. Roussel, Institut d'Astrophysique de Paris, CNRS, Univ. Pierre et Marie Curie (France); N. Schneider, Service d'Astrophysique, CEA (France); B. Schulz, A. Schwartz, D. L. Shupe, NASA Herschel Science Ctr. (United States); B. Sibthorpe, UK Astronomy Technology Ctr. (United Kingdom); S. Sidher, Rutherford Appleton Lab. (United Kingdom); A. J. Smith, Astronomy Ctr., Univ. of Sussex (United Kingdom); B. M. Swinyard, Rutherford Appleton Lab. (United Kingdom); M. Trichas, Imperial College London (United Kingdom); I. Valtchanov, European Space Astronomy Ctr. (Spain); A. L. Woodcraft, Institute for Astronomy, Univ. of Edinburgh (United Kingdom); C. K. XU, L. Zhang, NASA Herschel Science Ctr. (United States)

POSTER SESSION: HUBBLE

773138 WFC3 detectors: on-orbit performance [7731-113]

S. M. Baggett, J. W. MacKenty, Space Telescope Science Institute (United States);

R. A. Kimble, NASA Goddard Space Flight Ctr. (United States); T. Borders, B. Hilbert, S. Deustua, V. Kozhurina-Platais, K. S. Long, A. Riess, R. Gilliland, Space Telescope Science Institute (United States); R. J. Hill, Conceptual Analytics, LLC (United States); J. Kalirai, Space Telescope Science Institute (United States)

773139 Commissioning of the cosmic origins spectrograph on the Hubble Space Telescope: an overview of COS servicing mission observatory verification [7731-114]

D. J. Sahnow, The Johns Hopkins Univ. (United States); C. D. Keyes, T. B. Ake, A. Aloisi, Space Telescope Science Institute (United States); S. Béland, Univ. of Colorado at Boulder (United States); C. P. Biagetti, Space Telescope Science Institute (United States); E. B. Burgh, Univ. of Colorado at Boulder (United States); G. Chapman, Space Telescope Science Institute (United States); T. Delker, Ball Aerospace \& Technologies Corp. (United States); K. France, Univ. of Colorado at Boulder (United States); S. D. Friedman, Space Telescope Science Institute (United States); C. S. Froning, Univ. of Colorado at Boulder (United States); P. Ghavamian, P. Goudfrooij, Space Telescope Science Institute (United States); J. C. Green, Univ. of Colorado at Boulder (United States); G. F. Hartig, P. E. Hodge, D. Lennon, D. Massa, Space Telescope Science Institute (United States); J. B. McPhate, 
Space Sciences Lab., Univ. of California, Berkeley (United States); S.-M. Niemi, C. Oliveira, R. Osten, Space Telescope Science Institute (United States); S. N. Osterman, S. V. Penton, Univ. of Colorado at Boulder (United States); M. Reinhart, B. Shaw, T. E. Smith,

D. R. Soderblom, A. Welty, T. P. Wheeler, B. R. York, Space Telescope Science Institute (United States); W. Zheng, The Johns Hopkins Univ. (United States)

7731 3A HST/WFC3 in-orbit grism performance [7731-115]

H. Kuntschner, European Southern Observatory (Germany); H. Bushouse, Space Telescope Science Institute (United States); M. Kümmel, J. R. Walsh, European Southern Observatory (Germany); J. MacKenty, Space Telescope Science Institute (United States)

7731 3B Monitoring of the wavelength calibration lamps for the Hubble Space Telescope [7731-116] I. Pascucci, Space Telescope Science Institute (United States) and The Johns Hopkins Univ. (United States); C. Proffitt, Space Telescope Science Institute (United States) and Computer Sciences Corp. (United States); P. Ghavamian, Space Telescope Science Institute (United States); D. Sahnow, Space Telescope Science Institute (United States) and The Johns Hopkins Univ. (United States); C. Oliveira, A. Aloisi, T. Keyes, Space Telescope Science Institute (United States); S. V. Penton, Univ. of Colorado at Boulder (United States)

7731 3C Persistence and count-rate nonlinearity in the HST WFC3 IR detector [7731-117]

S. Deustua, K. S. Long, P. McCullough, Space Telescope Science Institute (United States); A. G. Riess, Space Telescope Science Institute (United States) and The Johns Hopkins Univ. (United States); J. MacKenty, Space Telescope Science Institute (United States); R. Kimble, NASA Goddard Space Flight Ctr. (United States); S. M. Baggett, B. Hilbert, Space Telescope Science Institute (United States); R. J. Hill, NASA Goddard Space Flight Ctr. (United States) and Conceptual Analytics, LLC (United States); C. Pavlovsky, L. D. Petro, Space Telescope Science Institute (United States)

\section{POSTER SESSION: INSTRUMENTS}

7731 3D Development and utilization of a point spread function for the Extrasolar Planet Observation and Characterization/Deep Impact Extended Investigation (EPOXI) Mission [7731-118] R. K. Barry, NASA Goddard Space Flight Ctr. (United States); D. Lindler, Sigma Space Corp. (United States); L. D. Deming, NASA Goddard Space Flight Ctr. (United States); M. F. A'Hearn, Univ. of Maryland, College Park (United States); S. Ballard, Harvard Univ. (United States); B. Carcich, Cornell Univ. (United States); D. Charbonneau, J. Christiansen, Harvard Univ. (United States); T. Hewagama, NASA Goddard Space Flight Ctr. (United States) and Univ. of Maryland, College Park (United States); L. McFadden, NASA Goddard Space Flight Ctr. (United States); D. Wellnitz, Univ. of Maryland, College Park (United States)

$77313 \mathrm{E}$ The ring of fire: an internal illumination system for detector sensitivity and filter bandpass characterization [7731-119]

V. E. Scarpine, S. M. Kent, Fermi National Accelerator Lab. (United States); S. E. Deustua, Space Telescope Science Institute (United States); M. J. Sholl, Lawrence Berkeley National Lab. (United States); S. L. Mufson, Indiana Univ. (United States); M. N. Ott, NASA Goddard Space Flight Ctr. (United States); M. P. Wiesner, Northern Illinois Univ. (United States); B. J. Baptista, Indiana Univ. (United States) 
$77313 \mathrm{~F}$ Monte Carlo simulations as a tool for radiation damage evaluation [7731-120]

S. Ligori, A. Riva, INAF, Osservatorio Astronomico di Torino (Italy); M. Mauri, European

Organization for Nuclear Research (Switzerland); L. Corcione, INAF, Osservatorio

Astronomico di Torino (Italy); F. Bortoletto, C. Bonoli, E. Giro, INAF, Osservatorio Astronomico

di Padova (Italy)

POSTER SESSION: JDEM

7731 3G An attitude control testbed for JDEM [7731-123]

K. Reil, A. Roodman, Kavli Institute for Particle Astrophysics and Cosmology, SLAC National Accelerator Lab. (United States); M. Sholl, Space Sciences Lab., Univ. of California, Berkeley (United States)

7731 3H A simple optical design for a space Dark Energy Mission [7731-124]

R. Grange, B. Milliard, J. P. Kneib, Lab. d'Astrophysique de Marseille (France); A. Ealet, Ctr. de Physique des Particules de Marseille (France)

7731 3l ACCESS: design and preliminary performance [7731-184]

M. E. Kaiser, J. W. Kruk, S. R. McCandliss, The Johns Hopkins Univ. (United States);

B. J. Rauscher, R. A. Kimble, NASA Goddard Space Flight Ctr. (United States); R. Pelton, D. J. Sahnow, W. V. Dixon, P. D. Feldman, B. W. Gaither, J. S. Lazear, H. W. Moos, The Johns Hopkins Univ. (United States); A. G. Riess, The Johns Hopkins Univ. (United States) and Space Telescope Science Institute (United States); D. J. Benford, J. P. Gardner, R. J. Hill, D. M. Kahle, D. B. Mott, A. Waczynski, Y. Wen, B. E. Woodgate, NASA Goddard Space Flight Ctr. (United States); R. C. Bohlin, S. E. Deustua, Space Telescope Science Institute (United States); R. Kurucz, Harvard-Smithsonian Ctr. for Astrophysics (United States); M. Lampton, Space Sciences Lab., Univ. of California, Berkeley (United States); S. Perlmutter, Univ. of California, Berkeley (United States); E. L. Wright, Univ. of California, Los Angeles (United States)

\section{Part Three}

\section{POSTER SESSION: JWST}

7731 3J The JWST/NIRCam coronagraph flight occulters [7731-125]

J. E. Krist, K. Balasubramanian, R. E. Muller, S. B. Shaklan, Jet Propulsion Lab. (United States); D. M. Kelly, Steward Observatory, The Univ. of Arizona (United States); D. W. Wilson, C. A. Beichman, E. Serabyn, Jet Propulsion Lab. (United States); Y. Mao, Lockheed Martin Space Systems Co. (United States); P. M. Echternach, J. T. Trauger, K. M. Liewer, Jet Propulsion Lab. (United States)

7731 3K Simulation and image reconstruction of IFU-spectrometer data from JWST-MIRI [7731-126] A. M. Glauser, Institute for Astronomy, ETH Zürich (Switzerland) and UK Astronomy Technology Ctr. (United Kingdom); A. Glasse, UK Astronomy Technology Ctr. (United Kingdom); J. E. Morrison, Steward Observatory, The Univ. of Arizona (United States); B. D. Kelly, M. Wells, UK Astronomy Technology Ctr. (United Kingdom); F. Lahuis, SRON Netherlands Institute for Space Research (Netherlands) and Leiden Observatory, Leiden Univ. (Netherlands); G. S. Wright, UK Astronomy Technology Ctr. (United Kingdom) 
$77313 \mathrm{~L}$ Characterization of the tunable filter imager etalon on the JWST Fine Guidance Sensor [7731-127]

C. S. Haley, C. Evans, E. Grant, R. Norman, Z. Osman, N. Rowlands, N. Roy, A. Scott,

D. Touahri, COM DEV Canada (Canada)

$77313 \mathrm{M}$ Compared sensitivities of VLT, JWST and ELT for direct exoplanet detection in nearby stellar moving groups [7731-128]

C. Hanot, O. Absil, J. Surdej, Institut d'Astrophysique et de Géophysique, Univ. de Liège (Belgium); A. Boccaletti, LESIA, Observatoire de Paris à Meudon (France); C. Vérinaud, Lab. d'Astrophysique de l'Observatoire de Grenoble (France)

$77313 \mathrm{~N} \quad$ Performance verification of the MIRI imager flight model at CEA [7731-129]

S. Ronayette, CEA, DSM, IRFU (France); C. Cavarroc, Spitzer Science Ctr., California Institute of Technology (United States); S. Kendrew, Leiden Observatory (Netherlands); J. Amiaux, J.-L. Auguères, M. Bouzat, CEA, DSM, IRFU (France); P. Guillard, Spitzer Science Ctr., California Institute of Technology (United States) and Institut d'Astrophysique Spatiale, CNRS, Univ. Paris 11 (France); V. Moreau, E. Pantin, P. Bouchet, A. Bensalem, T. Orduna, P.-O. Lagage, C. Nehme, A. Belu, CEA, DSM, IRFU (France); A. Glasse, UK Astronomy Technology Ctr. (United Kingdom); P. Baudoz, LESIA, Observatoire de Paris à Meudon (France); D. Dubreuil, CEA, DSM, IRFU (France)

773130 OGSE telescope WFE testing at 30K [7731-130] H. Lin, S. Delamer, C. Evans, A. McColgan, S. Zheng, P. Klimas, P. Marais, COM DEV International Ltd. (Canada); F. Grandmont, ABB Bomem Inc. (Canada)

$77313 \mathrm{P} \quad$ Optical wavefront characterization using phase retrieval for the NIRSpec demonstration model for the James Webb Space Telescope [7731-131]

J. S. Smith, D. L. Aronstein, P. S. Davila, B. H. Dean, NASA Goddard Space Flight Ctr. (United States); B. Dorner, CRAL, Observatoire de Lyon (France); X. Gnata, M. Melf, J.-F. Pittet, EADS Astrium GmbH (Germany); M. B. Te Plate, European Space Research and Technology Ctr. (Netherlands)

$77313 Q \quad$ Wavelength calibration of the JWST-MIRI medium resolution spectrometer [7731-132] J. R. Martínez-Galarza, Leiden Observatory, Leiden Univ. (Netherlands); A. M. Glauser, Institute of Astronomy, ETH Zurich (Switzerland) and UK Astronomy Technology Ctr. (United Kingdom); A. Hernán-Caballero, R. Azzollini, Consejo Superior de Investigaciones Científicas (Spain); A. Glasse, UK Astronomy Technology Ctr. (United Kingdom); S. Kendrew, B. Brandl, Leiden Observatory, Leiden Univ. (Netherlands); F. Lahuis, SRON Netherlands Institute for Space Research (Netherlands)

$77313 R \quad$ Speckle differential imagery performance using a JWST tunable filter etalon prototype [7731-134]

P. Ingraham, R. Doyon, M. Beaulieu, Univ. de Montréal (Canada); N. Rowlands, A. Scott, COM DEV Canada (Canada)

$773135 \quad$ MIRI-JWST spectrometer main optics flight model realization and performance test results [7731-135]

G. Kroes, A. Oudenhuysen, M. de Haan, ASTRON (Netherlands) and NOVA (Netherlands); R. Jager, NOVA (Netherlands); E. Pauwels, Pi Environments B.V. (Netherlands) 
7731 зт Use of a pathfinder optical telescope element for James Webb Space Telescope risk mitigation [7731-136]

L. D. Feinberg, R. Keski-Kuha, NASA Goddard Space Flight Ctr. (United States); C. Atkinson,

S. C. Texter, Northrop Grumman Aerospace Systems (United States)

$77313 \mathrm{U}$ Applying the tool: stray light cross-checks of the James Webb Space Telescope [7731-138] D. L. Skelton, Sigma Space Corp. (United States)

7731 3V Manufacturing and integration status of the JWST OSIM optical simulator [7731-139] J. Sullivan, Ball Aerospace \& Technologies Corp. (United States); B. Eichhorn, NASA Goddard Space Flight Ctr. (United States); R. von Handorf, D. Sabatke, N. Barr, R. Nyquist, B. Pederson, R. Bennnett, P. Volmer, D. Happs, A. Nagle, R. Ortiz, T. Kouri, P. Hauser, J. Seerveld, Ball Aerospace \& Technologies Corp. (United States); D. Kubalak, B. Greeley, C. Hakun, D. Leviton, Q. Gong, P. Davila, R. Ohl, NASA Goddard Space Flight Ctr. (United States); J. Kirk, C. Davis, J. Chu, Orbital Sciences Corp. (United States); E. Wilson, Stinger Ghaffarian Technologies (United States); B. Chang, Edge Space Systems, Inc. (United States); S. Mann, The Hammers Co. (United States); R. Rashford, Genesis Engineering Solutions, Inc. (United States); C. Smith, ATK Space Systems (United States)

7731 3W Planetary system and star formation science with non-redundant masking on JWST [7731-140]

A. Sivaramakrishnan, American Museum of Natural History (United States); D. Lafrenière, Univ. de Montréal (Canada); P. G. Tuthill, M. J. Ireland, The Univ. of Sydney (Australia); J. P. Lloyd, Cornell Univ. (United States); F. Martinache, Subaru Telescope, National Astronomical Observatory of Japan (United States); R. B. Makidon, R. Soummer, Space Telescope Science Institute (United States); R. Doyon, M. Beaulieu, Univ. de Montréal (Canada); S. Parmentier, Stony Brook Univ. (United States); C. A. Beichman, NASA ExoPlanet Science Institute, California Institute of Technology (United States)

\section{POSTER SESSION: MIRROR TECHNOLOGY}

$77313 X$ Development and tests of interferometry facility in 6-m diameter radiometer thermal vacuum chamber in Tsukuba Space Center [7731-142]

M. Suganuma, H. Katayama, M. Naitoh, T. Imai, M. Miyamoto, K. Maruyama, Japan Aerospace Exploration Agency (Japan); H. Kaneda, Nagoya Univ. (Japan); Y. Tange, T. Nakagawa, Japan Aerospace Exploration Agency (Japan)

7731 3Y ZERODUR 8m mirror for space telescope [7731-143]

P. Hartmann, T. Westerhoff, R. Reiter, R. Jedamzik, V. Wittmer, H. Kohlmann, SCHOTT AG (Germany)

\section{POSTER SESSION: NJASMINE}

$77313 Z$ Nano-JASMINE: current status and data output [7731-144]

Y. Kobayashi, National Astronomical Observatory of Japan (Japan) and The Univ. of Tokyo (Japan); T. Yano, National Astronomical Observatory of Japan (Japan); N. Gouda, National Astronomical Observatory of Japan (Japan) and The Univ. of Tokyo (Japan); Y. Niwa, J. Murooka, The Univ. of Tokyo (Japan); Y. Yamada, Kyoto Univ. (Japan); N. Sako,

S. Nakasuka, The Univ. of Tokyo (Japan) 
773141 CCD centroiding analysis for Nano-JASMINE observation data [7731-146]

Y. Niwa, T. Yano, H. Araki, N. Gouda, Y. Kobayashi, National Astronomical Observatory of Japan (Japan); Y. Yamada, Kyoto Univ. (Japan); S. Tazawa, H. Hanada, National

Astronomical Observatory of Japan (Japan)

\section{POSTER SESSION: SOLAR PLANETARY SCIENCE}

773142 The telescope and the double Fabry-Pérot interferometer for the ADAHELI solar space mission [7731-147]

V. Greco, CNR, Istituto Nazionale di Ottica Applicata (Italy); F. Cavallini, INAF, Osservatorio Astrofisico di Arcetri (Italy); F. Berrilli, Univ. degli Studi di Roma Tor Vergata (Italy)

773143 The thermo-optical design and experiment research on $\mathrm{H} \alpha$ and white light telescope [7731-148]

Z. Chen, M. Wu, S. Yang, X. Gu, S. Wang, National Astronomical Observatories (China)

773144 Simulation of the metrology of the PROBA-3/ASPIICS formation flying solar coronagraph [7731-149]

F. Stathopoulos, National Technical Univ. of Athens (Greece); A. Antonopoulos, Univ. of Athens (Greece); S. Vives, Lab. d'Astrophysique de Marseille, CNRS, Univ. de Provence (France); L. Damé, LATMOS/IPSL, CNRS, Univ. Versailles St-Quentin (France)

773145 The space instrument SODISM and the ground instrument SODISM II [7731-150] M. Meftah, M. Meissonnier, A. Irbah, S. Abbaki, LATMOS-IPSL, CNRS/INSU, Univ. Versailles St-Quentin (France); P. Assus, Observatoire de la Côte d'Azur, CNRS (France); E. Bertran, LATMOS-IPSL, CNRS/INSU, Univ. Versailles St-Quentin (France); J. P. Dubois, IAS, CNRS, Univ. Paris 11 (France); E. Ducourt, C. Dufour, J. P. Marcovici, G. Poiet, A. J. Vieau, G. Thuillier, LATMOS-IPSL, CNRS/INSU, Univ. Versailles St-Quentin (France)

773146 Stray light analysis and optimization of the ASPIICS/PROBA-3 formation flying solar coronagraph [7731-151]

A. Mazzoli, Ctr. Spatial de Liège (Belgium); F. Landini, Univ. degli Studi di Firenze (Italy); S. Vives, P. Lamy, Lab. d'Astrophysique de Marseille (France); J. P. Halain, P. Rochus, Ctr. Spatial de Liège (Belgium)

773147 Demonstrator of the formation flying solar coronagraph ASPIICS/PROBA-3 [7731-152] S. Vives, Lab. d'Astrophysique de Marseille, CNRS, Univ. de Provence (France); L. Damé, LATMOS/IPSL, CNRS, Univ. Versailles St-Quentin (France); P. Lamy, Lab. d'Astrophysique de Marseille, CNRS, Univ. de Provence (France); A. Antonopoulos, Univ. of Athens (Greece); W. Bon, Lab. d'Astrophysique de Marseille, CNRS, Univ. de Provence (France); G. Capobianco, G. Crescenzio, INAF, Osservatorio Astronomico di Torino (Italy); V. Da Deppo, IFN-CNR (Italy); M. Ellouzi, J. Garcia, C. Guillon, Lab. d'Astrophysique de Marseille, CNRS, Univ. de Provence (France); A. Mazzoli, Ctr. Spatial de Liège (Belgium); T. Soilly, Lab. d'Astrophysique de Marseille, CNRS, Univ. de Provence (France);

F. Stathopoulos, C. Tsiganos, Univ. of Athens (Greece) 
773148 Calibration and alignment of the demonstrator of the PROBA-3/ASPIICS formation flying coronagraph [7731-153]

G. Crescenzio, G. Capobianco, INAF, Osservatorio Astronomico di Torino (Italy);

V. Da Deppo, IFN-CNR (Italy); L. Damé, LATMOS/IPSL, CNRS, Univ. Versailles St-Quentin (France); S. Vivès, J. Garcia, C. Guillon, Lab. d'Astrophysique de Marseille, CNRS,

Observatoire Astronomique de Marseille-Provence (France)

773149 Analytic and experimental determination of ghosts in the Rosetta Narrow-Angle Camera and their impact on imaging performance [7731-154]

K. Dohlen, L. Jorda, P. Lamy, Lab. d'Astrophysique de Marseille, CNRS, Univ. de Provence (France); I. Toth, Konkoly Observatory (Hungary); A. Origne, Lab. d'Astrophysique de Marseille, CNRS, Univ. de Provence (France)

POSTER SESSION: SPICA

7731 4A Optical architecture of mid-infrared instruments (MIRACLE/MIRMES/MIRHES) on board SPICA [7731-155]

H. Kataza, T. Wada, Japan Aerospace Exploration Agency (Japan); Y. Ikeda, Kyoto-Sangyo Univ. (Japan); N. Fujishiro, Cybernet Systems Co., Ltd. (Japan); N. Kobayashi, I. Sakon, The Univ. of Tokyo (Japan)

$77314 \mathrm{~B}$ The digital processing unit of the SPICA SAFARI instrument: an FPGA based architecture using the Leon2-FT [7731-156]

A. M. Di Giorgio, F. Nuzzolo, D. Biondi, M. De Luca, P. Cerulli Irelli, R. Orfei, L. Spinoglio, INAF, Istituto di Fisica dello Spazio Interplanetario (Italy)

7731 4D Precision pointing control for SPICA: requirements and feasibility study [7731-158]

S. Mitani, T. Iwata, K. Fujiwara, S. Sakai, K. Enya, T. Kotani, H. Matsuhara, T. Nakagawa, Japan Aerospace Exploration Agency (Japan); T. Matsumoto, Seoul National Univ. (Korea, Republic of); W.-S. Jeong, D.-H. Lee, Korea Astronomy and Space Science Institute (Korea, Republic of)

7731 4E Polarization-interferometric eight-octant phase-mask coronagraph using ferroelectric liquid crystal for exoplanet detection [7731-159]

N. Murakami, T. Inabe, T. Komatsu, Hokkaido Univ. (Japan); J. Nishikawa, National Astronomical Observatory of Japan (Japan); N. Hashimoto, M. Kurihara, Citizen Holdings Co. Ltd. (Japan); N. Baba, Hokkaido Univ. (Japan); M. Tamura, National Astronomical Observatory of Japan (Japan)

$77314 \mathrm{~F}$ Development of a wavefront correction system for the SPICA coronagraph instrument [7731-160]

T. Kotani, K. Enya, T. Nakagawa, Japan Aerospace Exploration Agency (Japan); L. Abe, Univ. de Nice Sophia Antipolis (France); K. Haze, The Graduate Univ. for Advanced Studies (Japan); S. Higuchi, The Univ. of Tokyo (Japan); Y. Tange, Japan Aerospace Exploration Agency (Japan) 
7731 4G Conceptual design of a cryogenic system for the next-generation infrared space telescope SPICA [7731-161]

Y. Sato, H. Sugita, K. Shinozaki, A. Okamoto, T. Yamawaki, K. Komatsu, T. Nakagawa,

H. Murakami, H. Matsuhara, Japan Aerospace Exploration Agency (Japan); M. Murakami, Univ. of Tsukuba (Japan); M. Takada, S. Takai, A. Okabayashi, K. Kanao, S. Tsunematsu, K. Otsuka, K. Narasaki, Sumitomo Heavy Industries, Ltd. (Japan)

$77314 \mathrm{H} \quad$ Kinetic inductance detectors (KIDs) for the SAFARI instrument on SPICA [7731-195]

L. Ferrari, SRON National Institute for Space Research (Netherlands); A. M. Baryshev, SRON National Institute for Space Research (Netherlands) and Kapteyn Astronomical Institute, Univ. of Groningen (Netherlands); J. J. A. Baselmans, G. de Lange, P. Diener, SRON National Institute for Space Research (Netherlands); J. W. Kooi, California Institute of Technology (United States); J. J. Lankwarden, S. J. C. Yates, SRON National Institute for Space Research (Netherlands)

POSTER SESSION: STRATEGIES

773141 Overview of past and future space missions dedicated to exoplanet research [7731-162] L. Puig, S. Carpano, N. Rando, A. Stankov, I. Escudero, European Space Research and Technology Ctr. (Netherlands)

POSTER SESSION: SYSTEMS CONCEPTS

$77314 \mathrm{~J}$ An alternative architecture for the PLATO Mission [7731-164]

P. Laporte, G. Fasola, S. Mignot, Observatoire de Paris à Meudon, CNRS, Univ. Paris-Diderot (France)

7731 4K The PLATO opto-mechanical unit prototyping and AIV phase [7731-165] J. Farinato, INAF, Osservatorio Astronomico di Padova (Italy); V. Viotto, INAF, Osservatorio Astronomico di Padova (Italy) and Univ. degli Studi di Padova (Italy); G. Gentile, M. Dima, D. Magrin, INAF, Osservatorio Astronomico di Padova (Italy); D. Piazza, Univ. of Bern (Switzerland); R. Ragazzoni, INAF, Osservatorio Astronomico di Padova (Italy); G. Piotto, Univ. degli Studi di Padova (Italy); I. Pagano, INAF, Osservatorio Astrofisico di Catania (Italy); C. Arcidiacono, INAF, Osservatorio Astrofisico di Arcetri (Italy); S. Basso, INAF, Osservatorio Astronomico di Brera (Italy); W. Benz, Univ. of Bern (Switzerland); L. Gambicorti, Istituto Nazionale di Ottica Applicata (Italy); M. Ghigo, INAF, Osservatorio Astronomico di Brera (Italy); M. Munari, INAF, Osservatorio Astrofisico di Catania (Italy); E. Pace, Univ. degli Studi di Firenze (Italy); S. Scuderi, INAF, Osservatorio Astrofisico di Catania (Italy); C. Catala, Observatoire de Paris à Meudon (France)

7731 4L Achieving milli-arcsecond residual astrometric error for the JMAPS Mission [7731-169] G. S. Hennessy, U.S. Naval Observatory (United States); B. F. Lane, The Charles Stark Draper Lab., Inc. (United States); D. Veillette, C. Dieck, U.S. Naval Observatory (United States)

POSTER SESSION: TPF C

7731 4N Practical numerical propagation of arbitrary wavefronts through PIAA optics [7731-172] J. E. Krist, L. Pueyo, S. B. Shaklan, Jet Propulsion Lab. (United States) 
773140 A coronagraph system with unbalanced nulling interferometer: progress of wavefront correction [7731-173]

J. Nishikawa, National Astronomical Observatory of Japan (Japan); K. Yokochi, Tokyo Univ. of Agriculture and Technology (Japan) and National Astronomical Observatory of Japan (Japan); N. Murakami, Hokkaido Univ. (Japan); L. Abe, Univ. de Nice Sophia Antipolis (France); T. Kotani, Japan Aerospace Exploration Agency (Japan); M. Tamura, National Astronomical Observatory of Japan (Japan); T. Kurokawa, Tokyo Univ. of Agriculture and Technology (Japan); A. V. Tavrov, Space Research Institute (Russian Federation); M. Takeda, The Univ. of Electro-Communications (Japan); H. Murakami, Japan Aerospace Exploration Agency (Japan)

$77314 Q \quad$ CIAXE: co-axial achromatic interferential coronagraph: first laboratory results [7731-175] F. Allouche, ESO (Germany), Univ. de Nice Sophia-Antipolis, CNRS, Observatoire de la Côte d'Azur (France), and Collège de France, Chaire Astrophysique Observationnelle (France); J. Gay, Y. Rabbia, P. Assus, Univ. de Nice Sophia-Antipolis, CNRS, Observatoire de la Côte d'Azur (France)

7731 4S Progress on broadband control and deformable mirror tolerances in a 2-DM system [7731-177]

T. D. Groff, A. Carlotti, N. J. Kasdin, Princeton Univ. (United States)

$77314 \mathrm{~T}$ Studies of the effects of actuator errors on the HCIT/PIAA contrast performance [7731-179] E. Sidick, S. Shaklan, B. Kern, A. Give'on, Jet Propulsion Lab. (United States)

$77314 \mathrm{U}$ Low-cost high-precision PIAA optics for high contrast imaging with exo-planet coronagraphs [7731-180]

K. Balasubramanian, S. B. Shaklan, L. Pueyo, D. W. Wilson, Jet Propulsion Lab. (United States);

O. Guyon, Steward Observatory, The Univ. of Arizona (United States)

$77314 \mathrm{~V} \quad$ ACCESS pointing control system [7731-181]

P. Brugarolas, J. Alexander, J. Trauger, D. Moody, Jet Propulsion Lab. (United States);

R. Egerman, P. Vallone, J. Elias, ITT Corp. (United States); R. Hejal, V. Camelo, A. Bronowicki, D. O'Connor, R. Patrick, P. Orzechowski, C. Spittler, C. Lillie, Northrop Grumman Aerospace Systems (United States)

7731 4W Annular groove phase mask coronagraph in diamond for mid-IR wavelengths: manufacturing assessment and performance analysis [7731-183]

C. Delacroix, Univ. de Liège (Belgium); P. Forsberg, M. Karlsson, Uppsala Univ. (Sweden); D. Mawet, Jet Propulsion Lab. (United States); C. Lenaerts, Ctr. Spatial de Liège (Belgium); S. Habraken, Univ. de Liège (Belgium) and Ctr. Spatial de Liège (Belgium); C. Hanot, J. Surdej, Univ. de Liège (Belgium); A. Boccaletti, J. Baudrand, Observatoire de Paris à Meudon (France)

$77314 \mathrm{X}$ Simulations of coronagraphy with a dynamic hologram for the direct detection of exo-planets [7731-185]

D. Ricci, Univ. de Liège (Belgium); H. Le Coroller, Observatoire de Haute-Provence (France);

A. Labeyrie, Collège de France (France); P. Piron, Univ. de Liège (Belgium) 
$77314 \mathrm{Y}$ Design and implementation of the NUV/optical widefield Star Formation Camera for the Theia Observatory [7731-186]

P. A. Scowen, R. H. Jansen, Arizona State Univ. (United States); M. N. Beasley, Astrophysics Research Lab., Univ. of Colorado at Boulder (United States); D. Calzetti, Univ. of Massachusetts Amherst (United States); S. Desch, Arizona State Univ. (United States); A. W. Fullerton, Space Telescope Science Institute (United States); J. S. Gallagher III, Univ. of Wisconsin-Madison (United States); P. D. Lisman, S. A. Macenka, Jet Propulsion Lab. (United States); S. Malhotra, Arizona State Univ. (United States); M. J. McCaughrean, The Univ. of Exeter (United Kingdom); S. Nikzad, Jet Propulsion Lab. (United States); R. W. O'Connell, Univ. of Virginia (United States); S. Oey, Univ. of Michigan (United States); D. L. Padgett, Spitzer Science Ctr., California Institute of Technology (United States); J. E. Rhoads, Arizona State Univ. (United States); A. Roberge, NASA Goddard Space Flight Ctr. (United States); O. H. W. Siegmund, Space Science Lab,. Univ. of California, Berkeley (United States); S. B. Shaklan, Jet Propulsion Lab. (United States); N. Smith, Univ. of California, Berkeley (United States); D. Stern, Jet Propulsion Lab. (United States); J. Tumlinson, Space Telescope Science Institute (United States); R. A. Windhorst, Arizona State Univ. (United States); R. A. Woodruff, Lockheed Martin Space Systems Co. (United States)

$77314 Z$ Occulting ozone observatory ability to discover and locate single and multiple Earth-like planets in habitable zones [7731-187]

S. H. Pravdo, S. B. Shaklan, P. D. Lisman, Jet Propulsion Lab. (United States)

773150 Error analysis on the NWO starshade [7731-188]

T. Glassman, A. Johnson, A. Lo, D. Dailey, H. Shelton, J. Vogrin, Northrop Grumman Aerospace Systems (United States)

773151 Progress at the starshade testbed at Northrop Grumman Aerospace Systems: comparisons with computer simulations [7731-189]

R. Samuele, R. Varshneya, T. P. Johnson, A. M. F. Johnson, T. Glassman, Northrop Grumman Aerospace Systems (United States)

773152 Dynamical performance for science-mode stationkeeping with an external occulter [7731-190]

D. Sirbu, Princeton Univ. (United States); C. Vad Karsten, Princeton Univ. (United States) and DTU Space, Technical Univ. of Denmark (Denmark); N. J. Kasdin, Princeton Univ. (United States)

773153 Starshade design for occulter based exoplanet missions [7731-191]

M. W. Thomson, P. D. Lisman, R. Helms, P. Walkemeyer, A. Kissil, O. Polanco, Jet Propulsion Lab. (United States); S.-C. Lee, Applied Sciences Lab. (United States)

POSTER SESSION: WFSC

773154 First steps in the development of a piston sensor for large aperture space telescopes [7731-192]

G. Guerri, Ctr. Spatial de Liège (Belgium) and Institut d'Astrophysique et de Géophysique, Univ. de Liège (Belgium); S. Roose, Y. Stockman, A. Mazzoli, Ctr. Spatial de Liège (Belgium); J. Surdej, Institut d'Astrophysique et de Géophysique, Univ. de Liège (Belgium); J.-M. Defise, Ctr. Spatial de Liège (Belgium) 
773155 Advanced DFS: a dispersed fringe sensing algorithm insensitive to small calibration errors [7731-193]

J. A. Spechler, D. J. Hoppe, N. Sigrist, F. Shi, B.-J. Seo, S. Bikkannavar, Jet Propulsion Lab. (United States)

773156 False diamond turning artifacts in phase retrieval results [7731-194]

T. P. Zielinski, J. R. Fienup, The Institute of Optics, Univ. of Rochester (United States)

Author Index 


\title{
Conference Committee
}

\author{
Symposium Chairs
}

Masanori lye, National Astronomical Observatory of Japan (Japan)

Douglas A. Simons, Gemini Observatory (United States)

Symposium Cochairs

Mark M. Casali, European Organisation for Astronomical Research in the Southern Hemisphere (Germany)

Kathryn A. Flanagan, Space Telescope Science Institute (United States)

\section{Conference Chairs}

Jacobus M. Oschmann, Jr., Ball Aerospace \& Technologies Corporation (United States)

Mark C. Clampin, NASA Goddard Space Flight Center (United States)

Howard A. MacEwen, ManTech SRS Technologies (United States)

\section{Conference Cochairs}

Giovanni G. Fazio, Harvard-Smithsonian Center for Astrophysics (United States)

Takao Nakagawa, Japan Aerospace Exploration Agency (Japan)

\section{Program Committee}

Jonathan W. Arenberg, Northrop Grumman Aerospace Systems (United States)

James B. Breckinridge, College of Optical Sciences, The University of Arizona (United States)

Richard W. Capps, Jet Propulsion Laboratory (United States)

Suzanne Casement, Northrop Grumman Aerospace Systems (United States)

Lee D. Feinberg, NASA Goddard Space Flight Center (United States) James C. Green, University of Colorado at Boulder (United States) Matthew J. Griffin, Cardiff University (United Kingdom) Jean-Pierre Maillard, Institut d'Astrophysique de Paris (France) Gary M. Matthews, ITT Corporation (United States)

Mark J. McCaughrean, European Space Research and Technology Center (Netherlands)

David W. Miller, Massachusetts Institute of Technology (United States) Marc Postman, Space Telescope Science Institute (United States) Eric P. Smith, NASA Headquarters (United States) 
H. Philip Stahl, NASA Marshall Space Flight Center (United States)

Domenick J. Tenerelli, Lockheed Martin Space Systems Company (United States)

Wesley A. Traub, Jet Propulsion Laboratory (United States)

Gillian S. Wright, UK Astronomy Technology Centre (United Kingdom)

\section{Session Chairs}

1 Strategies

Eric P. Smith, NASA Headquarters (United States)

2 JWST I

Jacobus M. Oschmann, Jr., Ball Aerospace \& Technologies

Corporation (United States)

3 JWST II

Mark C. Clampin, NASA Goddard Space Flight Center (United States)

$4 \quad$ JWST III

Lee D. Feinberg, NASA Goddard Space Flight Center (United States)

5 AKARI/Spitzer

Jonathan W. Arenberg, Northrop Grumman Aerospace Systems (United States)

6 SPICA I

James B. Breckinridge, College of Optical Sciences, The University of Arizona (United States)

7 SPICA II

Takao Nakagawa, Japan Aerospace Exploration Agency (Japan)

8 WISE

Richard W. Capps, Jet Propulsion Laboratory (United States)

9 Hubble

Marc Postman, Space Telescope Science Institute (United States)

10 Herschel

Mark J. McCaughrean, European Space Research and Technology Center (Netherlands)

11 Kepler

James C. Green, University of Colorado at Boulder (United States) 
Howard A. MacEwen, ManTech SRS Technologies (United States)

13 GAIA

Suzanne Casement, Northrop Grumman Aerospace Systems (United States)

14 JDEM

Suzanne Casement, Northrop Grumman Aerospace Systems (United States)

15 Euclid

Jean-Pierre Maillard, Institut d'Astrophysique de Paris (France)

16 Systems Concepts I

H. Philip Stahl, NASA Marshall Space Flight Center (United States)

17 Systems Concepts II

Giovanni G. Fazio, Harvard-Smithsonian Center for Astrophysics (United States)

18 Systems Concepts III

Wesley A. Traub, Jet Propulsion Laboratory (United States)

19 Systems Concepts IV

Matthew J. Griffin, Cardiff University (United Kingdom)

20 TPF Coronagraph

Domenick J. Tenerelli, Lockheed Martin Space Systems Company

(United States)

21 TPF Occulter

Gillian S. Wright, UK Astronomy Technology Centre (United Kingdom)

22 ATLAST

Gary M. Matthews, ITT Corporation (United States)

23 Late Breaking News

Howard A. MacEwen, ManTech SRS Technologies (United States) 
Downloaded From: https://www.spiedigitallibrary.org/conference-proceedings-of-spie on 26 Apr 2023

Terms of Use: https://www.spiedigitallibrary.org/terms-of-use 


\section{Introduction}

This conference continues a SPIE biannual series' devoted to space-based astronomical sciences across the spectrum from the optical to the infrared, with occasional excursions into the millimeter wave region. As noted in this year's call for papers, 2010 looks to be a pivotal year for these sciences due particularly to the outcomes of two interrelated selection processes in Europe and the United States:

- Mission selection from the results of the European Space Agency (ESA) Cosmic Vision 2015-2025 Competitive Definition Phase, currently scheduled for 2010-2011 (and already well advanced)

- Recommendations of Astro2010: The Astronomy and Astrophysics Decadal Survey being conducted for the National Aeronautics and Space Administration (NASA) by the National Academy of Sciences and scheduled for release in September 2010.

Implementation of the results of these processes will, of course, be governed by multiple external factors, most importantly including:

- The ongoing global economic crisis, the resultant reduction of available resources, and the shifting personal and national priorities resulting from the crisis

- Possible competition from and/or synergies with the NASA manned exploration infrastructure that is continuing to undergo definition and refinement.

This Introduction is intended to identify and outline some of the most significant discussion threads that can be found in the papers. It does not pretend to follow the conference flow in detail or to summarize all important points found in the presentations. Emphasis is placed on the papers presented orally, but important supporting material was also presented throughout the poster sessions, and many of these manuscripts are also published in these proceedings.

\section{DEVELOPMENT AND ACQUISITION STRATEGIES}

The first session of the conference was devoted to papers addressing strategies relating to development of space telescope concepts in general. These specifically included realistic cost analysis; the availability and potential use of new enabling technologies; and possible benefits to be realized from systems designed for on-orbit servicing.

\footnotetext{
I Supplemented during alternate years by a similar but briefer conference at SPIE Optics+Photonics, the annual SPIE summer conference generally held in San Diego.
} 


\section{CURRENT CONSTELLATION}

Several sessions were devoted to updating the status and latest results from space telescopes presently in various phases of their operational lives. This was particularly timely, since many of the extant systems have either been launched or have entered new operational phases since the 2008 Marseille conference ${ }^{2}$. To be more specific:

- The Hubble Space Telescope (HST) received Servicing Mission 4 (SM4) in May 2009 that included the installation of two new instruments and repairs to the two other instruments that remained on the telescope, as well as maintenance for the spacecraft itself

- ESA launched the Herschel far infrared observatory3 in May 2009 as well. The vehicle has been successfully checked out and is now fully operational and producing results of great scientific merit

- Earlier in 2009 (March), NASA launched the Kepler observatory to search for transiting exoplanets, and has been rewarded with early and continuing success

- At the time of the conference, the NASA Wide Field Infrared Survey Explorer (WISE) was well into its ten month survey (launch occurred in December 2009) of the infrared sky which will provide a detailed foundation for future observational campaigns, most notably those of the James Webb Space Telescope (see below)

- Finally, results from two IR space telescopes currently operating in their warm modes (i.e., limited to the near-IR due to cryogen depletion) were reported. These telescopes are NASA's Spitzer (launched in 2003) and the Japanese AKARI (launched in 2006).

\section{JAMES WEBB SPACE TELESCOPE (JWST)}

JWST is clearly the flagship space telescope acquisition for the coming decade. Scheduled for launch in 2014, its many pieces are beginning to come together, perhaps most spectacularly the Optical Telescope Element (OTE), whose individual segments are completing polishing and testing in readiness for assembly. Several sessions were accordingly devoted to JWST and addressed multiple aspects of the total program, notably:

- Derivation of the science goals for JWST and flowdown to the specifications and design

- An overview of the complete development and acquisition program, including the measurement and testing programs

- A more focused overview of the development and fabrication of the OTE

- Detailed discussions of each of the science instruments as well as the Integrated Science Instrument Module (ISIM) as a whole

\footnotetext{
2 Astronomical Telescopes and Instrumentation 2008: Telescopes and Systems.

${ }^{3}$ Along with its companion Planck space telescope.
} 
- Specific, high priority foundational technologies (such as the wavefront sensing and control system) essential to enable the system.

\section{FUTURE SYSTEM POSSIBILITIES}

Approximately a third of the conference was devoted to consideration of possible future systems, including their science capabilities, system concepts, instrument designs and expected data output, and foundational technologies. We can expect that these potential systems will become the subjects of intense competition over the next year or two, particularly once the Decadal Survey recommendations have been published. Several thematic threads, mostly based upon significant science problems, are apparent in the papers.

- Planet finding, always with an underlying emphasis upon finding and characterizing terrestrial analogs, was addressed in a number of sessions. Four ${ }^{4}$ principal technical approaches were presented and discussed: astrometric measurements of stellar systems, planetary transits of the parent star, internal coronagraphs for direct viewing of exoplanets, and external occulters using a separate telescope and starshade some tens of thousands of kilometers from the telescope, also for direct viewing. Contributing subsystem technologies and testbeds were also considered, and there was a growing attention to concepts that would enable partial solutions (such as limiting attention to giant planets only, characterization of proto-planetary dust disks, and so on) while staying within severe fiscal constraints

- Investigation of dark energy provided a second area of major emphasis. A number of papers addressed general considerations for a Joint Dark Energy Mission (JDEM), but the majority of papers on this subject were specific to the ESA Euclid mission concept, specifically addressing the science requirements for the mission and the design and capabilities of proposed instruments. In general, concepts under consideration for the JDEM mission include instrumentation capable of addressing all three of the known scientific approaches to the study of dark energy: Baryonic Acoustic Oscillation, Weak Gravitational Lensing, and Type 1A Supernovae

- Two sessions were devoted to infrared astronomy that may be enabled by Japanese development of the Space IR Telescope for Cosmology and Astrophysics (SPICA). As in other cases, these sessions treated the science that could be realized with this observatory, provided an overview of the mission and system concept, and described a possible suite of instruments

- A small number of papers distributed throughout several different sessions 5 addressed technology and concepts for astrometry. This discipline contributes to almost all astrophysical topics: exoplanet detection and

\footnotetext{
${ }^{4}$ Use of a space-based interferometer for planet finding has been considered in the past, but no papers were presented on the topic at this Conference.

5 Based upon specific applications of the technology.
} 
mass determination (as noted above), cosmological mass distribution for dark matter and energy studies, and gravitational curvature of light for general relativity studies

- Finally, several papers presented throughout the conference discussed concepts for space telescopes that could be used for general purpose astronomy and astrophysics, notably in infrared spectral regions. These included both small (i.e., meter class apertures) and medium/large (4 meter class) systems, and several papers addressed approaches to increasing the affordability of such systems

- The conference concluded with a session addressing various aspects of the Advanced Technology Large Aperture Telescope (ATLAST) concept for a large UV to Near IR telescope at aperture sizes ranging from 8 meters to 16 meters or larger. Such a large general purpose telescope would have a wide range of possible applications, and would be capable of addressing almost all of the scientific issues discussed during the course of the conference.

\section{Jacobus M. Oschmann, Jr. Mark C. Clampin Howard A. MacEwen}

